


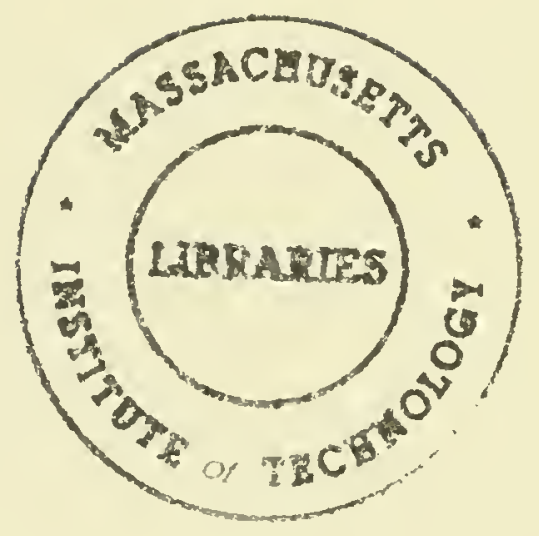




$\mathrm{HD} 28$

. $\mathrm{M} 414$

no.

$3367-$

92

\section{WORKING PAPER}

ALFRED P. SLOAN SCHOOL OF MANAGEMENT

OPTIMAL CONSUMPTION AND PORTFOLIO RULES WITH DURABILITY AND LOCAL SUBSTITUTION*

Ayman Hindy and Chi-fu Huang

November 1989

Revised November 1991

WP\# 3367-92-EFA

MASSACHUSETTS

INSTITUTE OF TECHNOLOGY

50 MEMORIAL DRIVE

CAMBRIDGE, MASSACHUSETTS 02139 



\section{OPTIMAL CONSUMPTION AND PORTFOLIO RULES WITH DURABILITY AND LOCAL SUBSTITUTION ${ }^{*}$ \\ Ayman Hindy and Chi-fu Huang \\ November 1989 \\ Revised November 1991}

WP\# 3367-92-EFA 



\title{
OPTIMAL CONSUMPTION AND PORTFOLIO RULES WITH DURABILITY AND LOCAL SUBSTITUTION*
}

\author{
Ayman Hindy \\ Graduate School of Business \\ Stanford University \\ Stanford, CA 94305 \\ and \\ Chi-fu Huang \\ Sloan School of Management \\ Massachusetts Institute of Technology \\ Cambridge, MA 02139
}

November 1989

Revised November 1991

\begin{abstract}
We study a model of optimal consumption and portfolio choice which captures, in two different interpretations, the notions of local substitution and irreversible purchases of durable goods. The class of preferences we consider excludes all nonlinear time-additive and nearly all the non-time-additive utility functions used in the literature. We discuss heuristically necessary conditions and provide sufficient conditions for a consumption and portfolio policy to be optimal. Furthermore, we demonstrate our general theory by solving in a closed form the optimal consumption and portfolio policy for a particular felicity function when the prices of the assets follow a geometric Brownian motion process. The optimal consumption policy in our solution consists of a possible initial "gulp" of consumption, or a period of no consumption, followed by a process of accumulated consumption with singular sample paths. In almost all states of nature, the agent consumes periodically and invests more in the risky assets than an agent with time-additive utility whose felicity function has the same curvature and the same time-discount parameter. We compute the equilibrium risk premium in a representative investor economy with a single physical production technology whose rate of return follows a Brownian motion. In addition, we provide some simulation results that demonstrate the properties of the purchase series for durable goods with different half-lives.
\end{abstract}

\footnotetext{
-We are grateful to Hua He for pointing out an error in an earlier version, and to Darrell Duffie, John Heaton, Jean-Luc Vila, Thaleia Zariphopoulou and anonymous referees for helpful comments. We acknowledge financial support from National Science Foundation under grants NSF-SES 9022937 and NSF SES-9022939
} 



\section{Introduction and Summary}

We study the problem of optimal consumption and portfolio choice for an agent whose preferences over consumption patterns are given by:

$$
\mathbf{E}\left[\int_{0}^{T} u(z(t), t) d t+V(W(T), z(T))\right]
$$

where

$$
z(t)=z\left(0^{-}\right) e^{-\beta t}+\beta \int_{0^{-}}^{t} e^{-\beta(t-s)} d C(s)
$$

and where $z(t)$ is the process of average past consumption derived from the consumption process $C(t)$ which denotes the total amount of consumption till time $t$. The parameter $\beta$ is a weighting factor, $z\left(0^{-}\right) \geq 0$ is a constant, and $W(T)$ is the random wealth left at time $T$. Both $u$ and $V$ are continuous and increasing functions. Furthermore, $V$ is concave and $u$ is concave in its first argument.

Two interesting economic notions are captured in two different interpretations of the model specified in (1) and (2). In one interpretation, preferences given by (1) embody the idea of local substitution, that is consumptions at nearby dates are almost perfect substitutes. In a second interpretation, the model represents preferences over the service flows from irreversible purchases of a durable good that decays over time.

Local substitution in continuous time, studied by Hindy, Huang and Kreps (1991) and Hindy and Huang (1991), is the notion that consumption at a point in time depresses marginal utility of consumption at nearby times. Suppression of appetite following a large meal is a natural phenomenon that leads to periodic consumption. In addition, local substitution implies that delaying or advancing consumption for a short period of time has little effect on satisfaction. In other words, agents regard consuming at adjacent dates as very similar alternatives.

Local substitution is absent in the nonlinear time-additive representations of preferences which are widely used in the finance and economics literature. Many researchers have recently used non-time-additive utilities; see, for example, Epstein (1987), Duffie and Epstein (1989), Epstein and $\operatorname{Zin}$ (1989), who study generalizations of the utility form introduced by Koopmans (1960) and Uzawa (1968) in the context of continuous time equilibrium models, and Constantinides (1990), Detemple and Zapatero (1990), Heaton (1990), and Sundaresan (1989) who study habit formation models.

The utility representation in (1) differs from most specifications in the non-time-additive preferences literature in one aspect which is very crucial in capturing the notion of local sub- 
stitution. In most of the currently used non-time-additive models, the index of instantaneous satisfaction, or felicity, at any time depends explicitly on the consumption rate at that time. Hindy, Huang and Kreps (1991) and Hindy and Huang (1991) show that when the felicity function depends explicitly on current consumption rates, the resulting preferences do not exhibit local substitution. In essence, the marginal utility for consumption at any moment is too sensitive to the current consumption rate and hence the agent would not tolerate delaying or advancing consumption even for a very small period of time. The utility function in (1), on the other hand, has the key feature that the felicity function at any time depends only upon an exponentially weighted average of past consumption - one derives satisfaction only from past consumption. This feature is the main difference between the utility function we study here and those studied by other researchers, except for Heaton $(1989,1990)$.

In the durable good version of the model, we interpret $C(t)$ as the total purchase of a durable good till time $t$. Purchases of the good are irreversible either because there is no secondary market for the good, or because of prohibitively high selling costs. The good provides a flow of services $z(t)$ at time $t$. Absent any new purchases, the service flow declines over time because of the deterioration in the stock of the durable good. Discrete time versions of this model have been studied by Dunn and Singleton (1986), Eichenbaum, Hansen and Singleton (1988) and Hotz, Kydland and Sedlacek (1988), among others. The model we study here can also be viewed as a limiting case of the model of Grossman and Laroque (1990) if we specify zero purchasing cost and infinite selling cost. We will henceforth use the terms "consumption" and "durable purchase" interchangeably.

The specification of utility in (1) leads to an economically interesting consumption behavior and also to a technically challenging optimization problem. The optimal consumption behavior for the agent with preferences given by (1) is periodic. The agent does not consume all the time as most models predict but only periodically. Most of the time the agent derives satisfaction from past consumption. When the marginal value of average past consumption, $z$, is higher than the marginal value of wealth, a situation that arises only periodically, the agent consumes. Otherwise, the agent refrains from consumption. In the durable good interpretation, the agent's purchases are periodic. Between purchases, the agent is content to use the service flow from the existing stock of the durable.

From the technical point of view, the standard dynamic programming equations of the Bellman-Jacobi type do not apply since a heuristic derivation of these equations would suggest a bang-bang control policy, but there is no natural upper bound on consumption. We follow a 
different approach to establish optimality.

We provide necessary and sufficient conditions for a consumption-portfolio policy to be optimal. We show that it is necessary and sufficient that the value function, or the maximum attainable utility starting from any state, satisfies a differential inequality which can be viewed as an application of the Bellman optimality principle. In particular, the conditions require that at all times the marginal value of wealth is at least as large as ( $\beta$ times) the marginal value of the average past consumption. Furthermore, consumption should only occur when these marginal values are equal.

We also construct a closed form solution of the optimal consumption problem for a particular class of felicity and bequeath functions in the case when the prices of the risky assets follow a geometric Brownian motion. The idea of the optimal solution is to keep the ratio $\frac{w}{z}$ less than a critical number, say $k^{*}$, and consume only when $\frac{W}{z}=k^{*}$. This requirement implies that consumption is periodic. Because of uncertainty about asset returns, however, an interesting phenomenon appears. The ratio $\frac{W}{z}$ is subject to random shocks because of the randomness of the return on the risky assets. Furthermore, the sample path properties of the Brownian motion lead to peculiar behavior of the quantity $\frac{W}{z}$ during the times when it is very close to the critical number $k^{*}$. The quantity $\frac{W}{z}$ fluctuates so fast that whenever it hits the value $k^{*}$ it bounces back and forth to hit it again uncountably infinite number of times. This results in a process of optimal cumulative consumption with nontrivial increasing sample paths in which consumption occurs only periodically.

We provide a complete solution for the optimal consumption and portfolio rules in this example and show how the solution changes with changes in the parameters of the problem. In particular, we compare the behavior of our agent to that of an agent with a time-additive utility whose felicity function has the same curvature and the same time-discount parameter. We show that the agent with local substitution preferences behaves in a less risk averse manner, in the sense that he invests higher proportions of his wealth in the risky assets. This behavior is reinforced by lower riskless rates, lower time-discount rate and higher effects of substitution (lower $\beta$ ).

It is worthwhile to mention that one obtains periodic consumption behavior in time additive models when the marginal felicity of consumption evaluated at zero is finite. In such a case, an agent with such preferences will refrain from consumption once his wealth falls below a critical, possibly time varying, level; see Cox and Huang (1989). In contrast, in our analysis, the optimal consumption is periodic, even when the felicity function has infinite slope at zero. In addition, 
in choosing consumption, the agent considers both current wealth and past consumption experience. Local substitution and durability produce important effects of past consumption and purchases on current utility.

We study the implication of the agent's behavior on the equilibrium in asset markets and on the determination of the risk premium. We show that the agent's portfolio choice implies two-fund separation and, hence, the Capital Asset Pricing Model holds. On the other hand, the Consumption Capital Asset Pricing Model fails to hold since the marginal utility of consumption is no longer connected to the marginal utility of wealth. Similar observations were made by Bergman (1985) and Grossman and Laroque (1990). We also compute the risk premium in equilibrium in the presence of a production technology with constant stochastic returns to scale. We show that the equilibrium risk premium will be lower in the case of local substitution, or durability, than in the case of time-additive utility. This result is consistent with the less risk averse behavior of the agents with local substitution or the case of durable goods.

We also study the properties of measured consumption and purchases over some fixed period using simulation. We construct temporal aggregates of purchases for a consumer who faces investment opportunities similar to the historical record of the U.S. stock market. For durable goods with different half-lives, we analyze the autocorrelations of the changes in measured purchases. We find that all autocorrelations are negative reflecting the durability of the consumption good. We also find that, for a fixed half-life, the longer the measurement period is, the smaller is the absolute value of the autocorrelation. This results from temporal aggregation. In addition, we find that, for any fixed measurement period, the longer is the half-life of the good, the higher is the absolute value of autocorrelation. This reflects the fact that the optimal policy for short-lived durables is to purchase small quantities frequently in contrast with the optimal policy for long-lived durables which calls for sporadic and large purchases. This finding also suggests that the time-series of purchases of good with short half-life will be "smoother" than that for a good with long half-life. The simulation results we report confirm this intuition.

The analysis of this paper suggests that local substitution, or durability, alone lead to lower risk premium on equity. Many researchers, most notably Constantinides (1990), have shown that habit formation leads to higher risk premium on equity. Habit formation, which is complementarity over longer periods, can be combined with local substitution, or durability, to produce interesting and rich behavior and asset price dynamics. The recent empirical evidence of Eichenbaum and Hansen (1990), Gallant and Tauchen (1989), and Heaton (1989, 1990) suggest that there is substitution over short periods. In particular, Heaton (1990) showed, 
after correcting for the problem of temporal aggregation, that habit formation alone does not provide significant explanation power for asset pricing over the time-additive models; while local substitution, or durability, does. In addition, given local substitution, or durability, habit formation over long horizons becomes more significant in its explanatory power. These results imply that a model which combines local substitution and habit formation is worth pursuing. In fact, this is the next step in this research program.

The analysis of this paper is focused on the decision of an individual to purchase a durable good. It is important to construct an aggregate series of purchases of durable goods and relate its dynamics to macroeconomic variables. More work needs to be done to achieve this objective. We refer the reader to Caballero (1991), and the references therein, for more on this important topic. The contribution of this analysis to the macroeconomics literature on durable goods is providing a closed form solution to the problem of an individual. This can serve as a concrete basis for further aggregation results.

The rest of this paper is organized as follows. Section 2 sets up the consumption and portfolio problem under uncertainty in continuous time. Section 3 provides a heuristic derivation of the necessary conditions for optimality, whereas Section 4 shows that these, together with some other regularity conditions, are sufficient. Section 5 provides a verification theorem for an infinite horizon program. In Section 6, we solve the optimal consumption and portfolio problem in closed form for a particular felicity function when the prices of risky assets follow a process of geometric Brownian motion. Section 7 provides some comparative statics and equilibrium implications and section 8 presents computations of the risk premium. The simulation results of measured, temporally aggregated, purchases appear in section 9 . Section 10 contains concluding remarks.

\section{Formulation}

Consider an agent who lives from time $t=0$ to $t=T$ in a world of uncertainty where there is a single good available for consumption at any time between 0 and $T$. The agent has the opportunity to invest in a frictionless securities market with $N+1$ long lived securities, continuously traded, and indexed by $n=0,1,2, \ldots, N$. Security $n$, where $n=1,2, \ldots, N$, is risky, pays dividends at rate $\rho_{n}(t)$, and sells for $S_{n}(t)$, at time $t$. We assume that $\rho_{n}(t)$ can be written as $\rho_{n}(S(t), t)$, where we have used $S(t)$ to denote the column vector $\left[S_{1}(t), S_{2}(t), \ldots, S_{N}(t)\right]^{\top}$.

\footnotetext{
${ }^{1}$ The superscript ${ }^{\top}$ denotes transpose.
} 
Security 0 is locally riskless, does not pay dividend, and sells for $B(t)=\exp \left\{\int_{0}^{t} r(S(s), s) d s\right\}$ at time $t$, where $r(S, t)$ is the instantaneous riskless interest rate at time $t$ and $r(\cdot, \cdot)$ is Borel measurable.

We will use the following notation: If $\mu$ is a vector in $\Re^{N}$, let $|\mu|$ be the Euclidean norm of $\mu$. In addition, if $\sigma$ is a matrix, let $|\sigma|^{2}$ denote $\operatorname{tr}\left(\sigma \sigma^{\top}\right)$, where $\operatorname{tr}$ is the trace of a square matrix.

The price process ${ }^{2}$ for the risky securities follows a diffusion process given by:

$$
S(t)+\int_{0}^{t} \rho(S(s), s) d s=S(0)+\int_{0}^{t} \mu(S(s), s) d s+\int_{0}^{t} \sigma(S(s), s) d B(s) \quad \forall t \in[0, T] \quad \text { a.s. }
$$

where $B$ is an $M$-dimensional, $M \geq N$, standard Brownian motion defined on a complete probability space $(\Omega, \mathcal{F}, P)$ and the notation a.s. denotes statements which are true with probability one. Assume that this diffusion process is strictly positive with probability one and that for each integer $m>0$, there exists a constant $c_{m}$ so that $E\left[|S(t)|^{2 m}\right] \leq e^{c_{m} t}{ }^{3}$. For brevity of notation we will sometimes use $\mu(t), \rho(t), \sigma(t)$, and $r(t)$ to denote $\mu(S(t), t), \rho(S(t), t)$, $\sigma(S(t), t)$ and $r(S(t), t)$, respectively.

We assume that the agent has only access to the information contained in the historical prices of the risky securities. We denote this information structure by $\mathbf{F}=\left\{\mathcal{F}_{t} ; t \in[0, T]\right\}$, where $\mathcal{F}_{t}$ is the smallest sub-sigma-field of $\mathcal{F}$ with respect to which $\{S(s) ; 0 \leq s \leq t\}$ is measurable. We assume that $\mathcal{F}_{t}$ contains all the probability zero sets of $\mathcal{F}$, or $\mathrm{F}$ is complete. All processes to be discussed will be adapted to $F^{4}$

The agent can consume the single good at "gulps" at any moment, and can consume at finite rates over intervals. The agent can also refrain from consumption altogether for some time. Moreover, the sample path of cumulative consumption at any time $t$ can have a singular component, that is a continuous nontrivial increasing function whose derivative is zero for almost all $t$.

Let $\mathrm{X}_{+}$be the space of all processes $x$ whose sample paths are positive, increasing and rightcontinuous. Recall that an increasing function $x(\omega,$.$) has a finite left-limit at any t \in(0, T]$ denoted by $x\left(\omega, t^{-}\right)$. We will use the convention that $x\left(\omega, 0^{-}\right)=0$ a.s. Since left limits exist for the sample paths of any $x \in \mathrm{X}_{+}$, a jump of $x(\omega,$.$) at \tau$ is $\Delta x(\omega, \tau) \equiv x(\omega, \tau)-x\left(\omega, \tau^{-}\right)$.

\footnotetext{
${ }^{2} \mathrm{~A}$ process $Y$ is a mapping $Y: \Omega \times[0, T] \rightarrow \Re$ that is measurable with respect to $\mathcal{F} \otimes B([0, T])$, the product sigma-field generated by $\mathcal{F}$ and the Borel sigma-field of $[0, T]$. For each $\omega \in \Omega, Y(\omega,):.[0, T] \rightarrow \Re$ is a sample path and for each $t \in[0, T], Y(., t): \Omega \rightarrow \Re$ is a random variable.

${ }^{3}$ The latter can be ensured by a growth condition on $(\mu-\rho)$ and on $\sigma$; see Friedman $(1975$, theorem 5.2.3).

'The process $Y$ is said to be adapted to $\mathbf{F}$ if for each $t \in[0, T], Y(t)$ is $\mathcal{F}_{t}$-measurable. This is a natural information constraint: the value of the process at time $t$ cannot depend on information yet to be revealed.
} 
The stochastic process $C \in \mathbf{X}_{+}$is a consumption pattern avajlable to the agent with $C(\omega, t)$ denoting the cumulative consumption from time 0 to time $t$ in state $\omega$. For any $\omega \in \Omega$, the points of discontinuity of $C(\omega, t)$ are the moments when the agent consumes a "gulp". Moreover, $C(\omega, t)$ has an absolutely continuous component over the intervals during which the agent is consuming at rates $C^{\prime}(\omega, t)$, where $C^{\prime}(\omega, t)$ denotes the consumption rate at time $t$ in state $\omega$. Finally, $C(\omega, t)$ may have a singular part. Singular components of consumption processes will play an important role in our analysis of the optimal consumption policy.

An investment strategy is an $N$-dimensional process $A=\left\{A(t) \equiv\left(A_{1}(t), \ldots, A_{N}(t)\right) ; t \in\right.$ $[0, T]\}$, where $A_{n}(t)$ denotes the proportion of wealth invested in the $n$-th risky security at time $t$ before consumption and trading. The proportion invested in the riskless security is $1-A(t)^{\top} 1$, where 1 is a vector of 1 's. A consumption plan $C \in X_{+}$is said to be financed by an investment strategy $A$ if

$$
\begin{aligned}
W(t)= & W(0)+\int_{0}^{t}\left(W(s) r(s)+W(s) A^{\top}(s) I_{S^{-1}}(s)(\mu(s)-r(s) S(s))\right) d s-C\left(t^{-}\right) \\
& +\int_{0}^{t} W(s) A^{\top}(s) I_{S^{-1}}(s) \sigma(s) d B(s) \quad \forall t \in[0, T] \quad \text { a.s. }
\end{aligned}
$$

where $W(t)$ is the wealth at time $t$ before consumption and $I_{S^{-1}}(t)$ is an $N \times N$ diagonal matrix with the $n$-th element on the diagonal being equal to $S(t)^{-1}$. Note that the wealth process has left-continuous sample paths and that $W\left(t^{+}\right)=W(t)-\Delta C(t)$.

The agent derives satisfaction from past consumption and from final wealth and utility is given by (1). The felicity function at time $t$ is defined over an exponentially weighted average of past consumption

$$
z(t)=z\left(0^{-}\right) e^{-\beta t}+\beta \int_{0^{-}}^{t} e^{-\beta(t-s)} d C(s) \text { a.s. }
$$

where $z\left(0^{-}\right) \geq 0$ is a constant, $\beta$ is a weighting factor, and the integral of $(5)$ is defined path by path in the Lebesgue-Stieltjes sense. Note that the lower limit of the integral in (5) is $0^{-}$, to account for the possible jump of $C$ at $t=0$, and that $z$ is a right continuous process which jumps whenever $C$ does. Moreover, $z$ has a singular component whenever $C$ does. Observe that higher values of $\beta$ imply higher emphasis on the recent past and less emphasis on consumption in the distant past. It has been shown by Hindy and Huang (1991) that this kind of preferences views consumption at nearby dates as close substitutes: delaying or advancing consumption for a very small period of time, without violating the condition that consumption at $t$ depends only on information available up to $t$, has a very small effect on the agent's total satisfaction. 
A consumption plan $C$ and the investment strategy $A$ that finances it are said to be admissible if (4) is well-defined ${ }^{5}$, and for all integers $m>0$, there exists $k_{m}$ so that, for all $t$

$$
\begin{aligned}
E\left[|C(t)|^{2 m}\right] & \leq e^{k_{m} t} \\
E\left[|W(t)|^{2 m}\right] & \leq e^{k_{m} t}
\end{aligned}
$$

Denote by $\mathcal{C}$ and $\mathcal{A}$ the space of admissible consumption plans and trading strategies, respectively.

Formally, the agent manages wealth dynamically to solve the following program:

$$
\begin{array}{cl}
\sup _{C \in \mathcal{C}} & E\left[\int_{0}^{T} u(z(t), t) d t+V\left(W\left(T^{+}\right), z(T)\right)\right] \\
\text { s.t. } & C \text { is financed by } A \in \mathcal{A} \text { with } W(0)=W_{0}, \\
& \text { and } W(t)-\Delta C(t) \geq 0 \quad \forall t \in[0, T],
\end{array}
$$

where $W_{0}$ is the initial wealth of the agent, $u(\cdot, t)$ is the felicity function at time $t$, and $V$ is the felicity function for final wealth and terminal stock of the durable good. Note that the second constraint of $(7)$ is a positive wealth constraint - wealth after consumption at any time must be positive.

One objective of this paper is to derive necessary and sufficient conditions for a consumption pattern $C \in \mathcal{C}$ financed by some $A \in \mathcal{A}$ to be optimal. To achieve this end, we will also consider the utility maximization problem of the agent at time $t \geq 0$ given that wealth is $W$ and the exponentially weighted past consumption is $z$, both before consumption at that time. We define $^{6}$

$$
\begin{aligned}
J(W, z, S, t) \equiv \sup _{C \in \mathcal{C}_{t}} & E_{t}\left[\int_{t}^{T} u(z(s), s) d s+V\left(W\left(T^{+}\right), z(T)\right)\right] \\
\text { s.t. } & C \text { is financed by } A \in \mathcal{A}_{t} \text { with } W(t)=W, \\
& \text { and } W(s)-\Delta C(s) \geq 0 \quad \forall s \in[t, T],
\end{aligned}
$$

where $\mathcal{C}_{t}$ and $\mathcal{A}_{t}$ are the spaces of admissible consumption plans and trading strategies starting from $t$ with the convention that $C_{t^{-}}=0$ for all $C \in \mathcal{C}_{t}$, the admissibility is defined by (6) with $t$ replaced by $s$, for all $s \in[t, T]$, and

$$
z(s)=z e^{-\beta(s-t)}+\beta \int_{t^{-}}^{s} e^{-\beta(v-t)} d C(v)
$$

\footnotetext{
${ }^{3}$ For this we mean both the Lebesgue integral and the Ito integral are well-defined. When $A(t)$ is a feedback control depending on $\left(W(t), z\left(t^{-}\right), S(t), t\right)$ and $C(t)$ depends on the history of $(W, S)$, we mean there exists a solution $W$ to the stochastic differential equation (4).

${ }^{6} E_{\mathrm{t}}$ denotes expectation conditional on $\mathcal{F}_{\mathfrak{t}}$.
} 
Note that $J\left(W(t), z\left(t^{-}\right), S(t), t\right)$ is the agent's optimal expected life time utility at time $t$ before consumption purchases at that time and $J\left(W\left(t^{+}\right), z(t), S(t), t\right)$ is the optimal expected life time utility after the consumption purchases at time $t$. Often, we will refer to $J$ as the value function.

Note that if $C$ is a solution to (7) financed by $A$, then $C_{t} \equiv\left\{C(s)-C\left(t^{-}\right) ; s \in[t, T]\right\}$ is a solution to (8) financed by $A$ restricted to $[t, T]$.

\section{Heuristic Derivation of Necessary Conditions}

We will use Bellman's optimality principle to derive necessary conditions about $J$ assuming that it has certain smoothness properties. One difficulty that arises in this endeavor is that the usual Bellman equation in dynamic programming is derived when the admissible consumption plans are purely at rates and are feedback controls. Here, cumulative consumption can be in gulps and may have singular parts. Moreover, if the cumulative consumption has singular components, it cannot be expressed in feedback form. Thus, our derivation of the necessary conditions will be heuristic in nature.

First, we observe that if a consumption gulp of size $\Delta$ is prescribed at time $t$ when $W(t)=W$, $z\left(t^{-}\right)=z$, and $S(t)=S$, we must have

$$
J(W, z, S, t)=J(W-\Delta, z+\beta \Delta, S, t) .
$$

This is so because both quantities are equal to

$$
E_{t}\left[\int_{t}^{T} u(z(s), s) d s+V\left(W\left(T^{+}\right), z(T)\right)\right],
$$

where $\{z(s) ; s \in[t, T]\}$ and $W\left(T^{+}\right)$are defined along the optimal path on $[t, T]$. Moreover, the size of the gulp should be chosen to maximize $J$. Thus, we must have

$$
J_{W}(W-\Delta, z+\beta \Delta, S, t)=\beta J_{z}(W-\Delta, z+\beta \Delta, S, t),
$$

where $J_{W}$ and $J_{z}$ denote the first partial derivatives of $J$ with respect to its first and second arguments, respectively.

We now show that (9) and (10) imply that $J_{W}$ must be equal to $\beta J_{z}$ at any $(W, z, S, t)$ where a gulp of consumption is prescribed. Differentiating (9) with respect to $W$ while noticing that $\Delta$ is an implicit function of $W$ and $z$ defined through (10) gives

$$
\begin{aligned}
J_{W}(W, z, S, t)= & {\left[-J_{W}(W-\Delta, z+\beta \Delta, S, t)+\beta J_{z}(W-\Delta, z+\beta \Delta, S, t)\right] \frac{\partial \Delta}{\partial W} } \\
& \quad+J_{W}(W-\Delta, z+\beta \Delta, S, t) \\
= & J_{W}(W-\Delta, z+\beta \Delta, S, t),
\end{aligned}
$$


where we have used (10) to get the second equality. Similarly, we have $J_{z}(W, z, S, t)=J_{z}(\mathrm{IW}-$ $\Delta, z+\beta \Delta, S, t)$. Given (10), we then have

$$
J_{W}(W, z, S, t)=\beta J_{z}(W, z, S, t)
$$

at $(W, z, S, t)$ where a consumption gulp is prescribed.

Second, assume that $J$ is sufficiently smooth for the generalized Itô's lemma to apply. ${ }^{7}$ For any time $t<T$, the principle of optimality in dynamic programming and the generalized Itó's lemma imply that

$$
\begin{aligned}
0= & \max _{d C, A} E_{t}\left[\int_{t}^{t+\Delta} u(z(s), s) d s+\int_{t}^{t+\Delta}\left[\mathcal{D}^{A} J(s)+J_{s}(s)\right] d s\right. \\
& +\int_{t^{-}}^{t+\Delta}\left[\beta J_{z}(s)-J_{W}(s)\right] d C(s)+\sum_{i}\left[J\left(\tau_{i}^{+}\right)-J\left(\tau_{i}\right)\right]-\sum_{i}\left[J_{W}\left(\tau_{i}\right) \Delta W\left(\tau_{i}\right)\right. \\
& \left.\left.+J_{z}\left(\tau_{i}\right) \Delta z\left(\tau_{i}\right)\right]\right] \quad \text { a.s., }
\end{aligned}
$$

where $d C$ denotes the consumption plan on the time interval $[t, t+\Delta), \tau_{i}$ is the $i$-th jump point prescribed by $d C$ on $[t, t+\Delta), J(s)$ and its derivatives are evaluated at $\left(W(s), z\left(s^{-}\right), S(s), s\right)$,

${ }^{7}$ The generalized Itó lemma we will use throughout can be found in Krylov (1980, theorem 2.10.1) and Dellacherie and Meyer (1982, VIII.27): Fix a consumption policy financed by a trading strategy and define the proportion invested in risky securities $A(t)$ accordingly. Let $S(t), W(t), z(t)$ follow the dynamics in (3), (4) and (5), respectively. Let $f(W, z, S, t): \Re^{N+2} \times[0, T] \rightarrow \Re$ be once continuously differentiable on $\Re^{N+2} \times[0, T]$ in all of its arguments and twice continuously differentiable on $\Re^{N+2} \times[0, T]$ in its first $N+2$ arguments, except possibly on a smooth manifold $\mathcal{M}$. (A smooth manifold $\mathcal{M}$ in $\Re^{N+2} \times[0, T]$ is given by: $\mathcal{M}=\{(W, z, S, t) \in$ $\left.\Re^{N+2} \times[0, T]: \phi_{l}(W, z, S, t)=0, \quad l=1,2, \ldots, L\right\}$, where $\phi_{l}(W, z, S, t): \Re^{N+2} \times[0, T]-\Re, l=1,2, \ldots, L$, with $L \leq N+3$, is continuously differentiable in all its arguments.) Then, for all optional times $t \leq T$, we have:

$$
\begin{aligned}
f\left(W\left(t^{+}\right), z(t), S(t), t\right)= & f\left(W(0), z\left(0^{-}\right), S(0), 0\right)+\int_{0}^{t}\left[\mathcal{D}^{A} f(s)+f_{s}(s)\right] d s \\
& +\int_{0}^{t}\left[f_{W}(s) W(s) A(s)^{\top} I_{S^{-1}}(s) \sigma(s)+f_{S}(s)^{\top} \sigma(s)\right] d B(s) \\
& +\int_{0-}^{t}\left(\beta f_{z}(s)-f W(s)\right) d C(s) \\
& +\sum_{1} \Delta f\left(r_{i}\right)-\sum_{1}\left[f W\left(\tau_{1}\right) \Delta W\left(\tau_{1}\right)+f_{z}\left(\tau_{1}\right) \Delta z\left(\tau_{1}\right)\right] \text { a.s. }
\end{aligned}
$$

where $\tau_{1}, \tau_{2}, \ldots$, are the jump points of $C$ on $[0, t]$, where all the partial derivatives are evaluated at the points $\left(W(t), z\left(t^{-}\right), S(t), t\right)$, where $\mathcal{D}^{A} f$ is the differential generator of $f$ associated with the trading strategy and is given by:

$$
\begin{aligned}
\mathcal{D}^{A} f= & f_{W}\left[W r+W A^{\top} I_{S^{-1}}(\mu-r S)\right]-f_{z} \beta z+f_{S}^{\top}(\mu-\rho) \\
& +\frac{1}{2}\left[f_{W W} W^{2}\left(A^{\top} I_{S-1} \sigma\right)\left(A^{\top} I_{S-1} \sigma\right)^{\top}\right. \\
& \left.+2 f_{W S}^{\top}\left(W \sigma \sigma^{\top} I_{S-1} A\right)+\operatorname{tr}\left(f_{S S} \sigma \sigma^{\top}\right)\right] .
\end{aligned}
$$

and where $\Delta f\left(\tau_{1}\right)=f\left(W\left(\tau_{1}^{+}\right), z\left(\tau_{1}\right), S\left(\tau_{1}\right), \tau_{1}\right)-f\left(W\left(\tau_{1}\right), z\left(\tau_{1}^{-}\right), S\left(\tau_{1}\right), \tau_{1}\right)$. 


$$
\begin{aligned}
& \lim _{t \uparrow T} J(W, z, S, t)=V(W, z), \\
& \lim _{W \downarrow 0} J(W, z, S, t)=\int_{t}^{T} u\left(z e^{-\beta(s-t)}, s\right) d s+V\left(0, z e^{-\beta(T-t)}\right) .
\end{aligned}
$$

The first boundary condition is implied by the fact that any optimal consumption plan must involve no gulps at time $T$ since such a gulp cannot contribute to the agent's satisfaction from increases in $z$ and, furthermore, it reduces the terminal wealth. The second condition is implied by the constraint that wealth at any time cannot become negative. Thus, whenever wealth is zero, the only feasible policy afterwards is no consumption.

\section{Sufficiency}

In this section we provide a verification theorem for optimal controls. We will proceed with two steps. First, we show that if there exists a solution $\hat{J}$ to the differential inequality (14) with the two boundary conditions (15) and (16), and $\hat{J}$ satisfies some regularity conditions, then $\hat{J}(W, z, S, t) \geq J(W, z, S, t)$ for all $(W, z, S, t)$. Second, we then give conditions so that $\hat{J}(W, z, S, t)$ is attained by a candidate feasible investment and consumption policy. It then follows that $J(W, z, S, t)=\hat{J}(W, z, S, t)$ and the candidate investment and consumption policy is the optimal policy.

Proposition 1 Let there be a differentiable function $\hat{J}(Y, t):(0, \infty)^{N+2} \times[0, T) \rightarrow \nVdash$, concave in its first two arguments, which is continuously differentiable in all of its arguments, and twice continuously differentiable in its first $N+2$ arguments, except possibly on a smooth manifold $\mathcal{M},{ }^{8}$ satisfying the differential inequality (14) in the interior of its domain with boundary conditions (15) and (16). In addition, let $u$ and $\hat{J}$ satisfy the growth conditions: there exist $H_{1}>0$ and $\mathrm{I}_{2}>0$

$$
\begin{aligned}
& |u(x, t)| \leq K_{1}(1+|x|)^{K_{2}} \quad \forall x \in \Re_{+}, t \in[0, T], \\
& |\hat{J}(Y, t)| \leq K_{1}(1+|Y|)^{K_{2}} \quad \forall Y \in R_{+}^{N+2}, t \in[0, T],
\end{aligned}
$$

Then, for all $t \in[0, T]$,

$$
\mathrm{E}_{t}\left[\int_{t}^{T} u(z(s), s) d s+V\left(W\left(T^{+}\right), z(T)\right)\right] \leq J\left(W(t), z\left(t^{-}\right), S(t), t\right) \leq \hat{J}\left(W(t), z\left(t^{-}\right), S(t), t\right),
$$

\footnotetext{
${ }^{8}$ For the definition of a smooth manifold see footnote 7 .
} 
for all $C \in \mathcal{C}_{t}$ financed by some $A \in \mathcal{A}_{t}$, where $z(s)$ and $W(s), s \in[t, T]$, are the average past consumption and wealth, respectively, associated with $C$ and $A$.

Proof. See appendix.

Now, if there exists, for any time $t \leq T$ and given $\left(W(t), z\left(t^{-}\right), S(t), t\right)$, a feasible consumption plan $C^{*} \in \mathcal{C}_{t}$ financed by $A^{*} \in \mathcal{A}_{t}$ so that

$$
j\left(W(t), z\left(t^{-}\right), S(t), t\right)=E_{t}\left[\int_{t}^{T} u\left(z^{*}(s), s\right) d s+V\left(W^{*}\left(T^{+}\right), z^{*}(T)\right)\right],
$$

where $z^{*}$ and $W^{*}$ denote the weighted average past consumption and the wealth associated with $\left(C^{*}, A^{*}\right)$, respectively, then it must be the case that $\hat{J}\left(W(t), z\left(t^{-}\right), S(t), t\right)=J\left(W(t), z\left(t^{-}\right), S(t), t\right)$, and $\left(C^{*}, A^{*}\right)$ is the optimal consumption and investment policy starting from the $\left(W(t), z\left(t^{-}\right), S(t), t\right)$.

The following is the main theorem of this section, which provides conditions for a candidate consumption and investment policy to be optimal.

Theorem 1 Let $\hat{J}$ satisfy the conditions of Proposition 1. Assume furthermore that, starting at any time $t \in[0, T]$, with $W(t), z\left(t^{-}\right), S(t)$, there exists a consumption policy $C^{*} \in \mathcal{C}_{t}$ financed by $A^{*} \in \mathcal{A}_{t}$ with the associated state variables $W^{*}$ and $z^{*}$, such that, putting $\varrho=\inf \{s \geq t$ : $\left.W^{*}(s)=0\right\}$,

$$
\begin{aligned}
u\left(z^{*}, s\right)+\mathcal{D}^{A^{*}} \hat{J}+\hat{J}_{s} & =0 \quad \forall \text { a.e. } s \in(t, \varrho) \text { a.s. } \\
\int_{t}^{s}\left[\hat{J}_{W}\left(W^{*}, z^{*}, S, v\right)-\beta \hat{J}_{z}\left(W^{*}, z^{*}, S, v\right)\right] d C^{*}(v) & =0 \quad \forall s \in(t, \varrho] \text { a.s. }
\end{aligned}
$$

and

$$
\hat{J}\left(W\left(t_{i}\right), z\left(t_{i}^{-}\right), S\left(t_{i}\right), t_{i}\right)-\hat{J}\left(W\left(t_{i}\right)-\Delta C^{*}\left(t_{i}\right), z\left(t_{i}^{-}\right)+\beta \Delta C^{*}\left(t_{i}\right), S\left(t_{i}\right), t_{i}\right)=0 \quad a . s .
$$

where $t_{i}$ 's are the times of gulps prescribed by $C^{*}$ on $[t, \varrho)$. Then $\hat{J}\left(W(t), z\left(t^{-}\right), S(t), t\right)=$ $J\left(W(t), z\left(t^{-}\right), S(t), t\right)$ and $\left(C^{*}, A^{*}\right)$ is an optimal consumption and investment policy starting at $t$.

Proof. See appendix.

As a recipe for decision making, theorem 1 outlines general principles that the agent should follow as long as his wealth is strictly positive. In particular, the theorem instructs the agent to use a control policy that keeps the pair $(W, z)$ in the region where the differential equation (19) is satisfied. In addition, the agent may consume only when the marginal utility of wealth 
is equal to ( $\beta$ times) the marginal utility of the service flow. Finally, in the contingency that the optimal consumption policy calls for a "gulp", the size of the gulp should be chosen such that the value function immediately before is equal to the value function immediately after the gulp.

The optimal consumption and portfolio policy characterized in Theorem 1 of course satisfies all the necessary conditions of Section 3.

\section{A Verification Theorem for the Infinite Horizon Program}

The necessary and sufficient conditions for optimality presented in the above two sections are for the finite horizon program of ( 7 ). In this section, we will let $T=\infty$ and $V=0$ in ( 7 ) and replace the admissibility conditions of (6) for $C$ financed by $A$ by:

$$
E\left[\int_{0}^{\infty}|u(z(s), s)| d s\right]<\infty
$$

where $z$ is associated with $C$, and for all integers $m>0$ and $T \in \Re_{+}$, there exists $k_{m}^{T}$ so that, for all $t \leq T$,

$$
\begin{aligned}
E\left[|C(t)|^{2 m}\right] & \leq e^{k_{m}^{T} t}, \\
E\left[|W(t)|^{2 m}\right] & \leq e^{k_{m}^{T} t} .
\end{aligned}
$$

Similarly let $\mathcal{C}$ and $\mathcal{A}$ denote the space of admissible consumption policies and investment policies starting at $t=0$, respectively. In addition, $\mathcal{C}_{t}$ and $\mathcal{A}_{t}$ are the corresponding admissible spaces starting at $t$.

Consider the infinite horizon program:

$$
\begin{array}{rl}
J(W, z, S, t) \equiv \sup _{C \in \mathcal{C}_{s}} & E\left[\int_{t}^{\infty} u(z(s), s) d s \mid \mathcal{F}_{t}\right] \\
\text { s.t. } & C \text { is financed by } A \in \mathcal{A}_{t} \text { with } W(t)=W, \\
& \text { and } W(t)-\Delta C(s) \geq 0 \quad \forall s \geq t .
\end{array}
$$

We record below a verification theorem for this infinite horizon program:

Theorem 2 Let $\hat{J}: \Re_{+}^{N+3} \rightarrow \Re_{+}$be positive, concave in its first two arguments, continuously differentiable over $\Re_{+}^{N+3}$ in all of its arguments, and twice continuously differentiable over $\Re_{+}^{N+3}$ in its first $N+2$ arguments, except possibly on a smooth manifold $\mathcal{M}$, satisfying the differential inequality of (14) with the boundary condition

$$
\lim _{W \downarrow 0} \hat{J}(W, z, S, t)=\int_{t}^{\infty} u\left(z e^{-\beta(s-t)}, s\right) d s<\infty .
$$


In addition, $\hat{J}$ satisfies the growth condition: for every $T \in \Re_{+}$, there exists $K_{1}^{T}>0$ and $H_{2}^{-T}>0$ so that

$$
|\hat{J}(Y, t)| \leq K_{1}^{T}(1+|Y|)^{k_{2}^{T}} \quad \forall Y \in R_{+}^{N+2}, t \in[0, T] .
$$

Assume furthermore that, starting at any time $t<\infty$ with $W(t), z\left(t^{-}\right), S(t)$, there exists a consumption policy $C^{*} \in \mathcal{C}_{t}$ financed by $A^{*} \in \mathcal{A}_{t}$, with the associated state variables $W^{*}$ and $z^{*}$, such that, putting $\varrho=\inf \left\{s \geq t: W^{*}(s)=0\right\}$,

$$
\begin{aligned}
u\left(z^{*}, s\right)+\mathcal{D}^{A^{*}} \hat{J}+\hat{J}_{s} & =0 \quad \forall \text { a.e.s } \in(t, \varrho) \text { a.s. } \\
\int_{t}^{s}\left[\hat{J}_{W}\left(W^{*}, z^{*}, S, v\right)-\beta \hat{J}_{z}\left(W^{*}, z^{*}, S, v\right)\right] d C^{*}(v) & =0 \quad \forall s \in(t, \varrho] \text { a.s. } \\
\lim _{s \rightarrow \infty} E_{t}\left[\hat{J}\left(W^{*}(t+s), z^{*}\left(t+s^{-}\right), S(t+s), t+s\right)\right] & =0, \quad \text { a.s. }
\end{aligned}
$$

and

$$
\hat{J}\left(W^{\prime}\left(t_{i}\right), z\left(t_{i}^{-}\right), S\left(t_{i}\right), t_{i}\right)-\hat{J}\left(W\left(t_{i}\right)-\Delta C^{*}\left(t_{i}\right), z\left(t_{i}^{-}\right)+\beta \Delta C^{*}\left(t_{i}\right), S\left(t_{i}\right), t_{i}\right)=0 \quad a . s .,
$$

where $t_{i}$ 's are the times of gulps prescribed by $C^{*}$ on $[t, \varrho)$. Then $\hat{J}=J$ and $\left(C^{*}, A^{*}\right)$ is an optimal consumption and investment policy starting at $t$.

In the infinite horizon program, two more conditions are added. First, $\hat{J}$ is positive. Second, the expected value of $\hat{J}(t)$ along the optimal path must converge to zero as $t$ increases to infinity. The latter condition ensures that the agent exhibits enough impatience so that accumulating wealth indefinitely without consumption is not optimal. We imposed the former condition for technical convenience and we can replace it by a stronger condition which requires that (28) holds not just for the optimal policy but for all feasible plans.

\section{A Closed Form Solution for an Infinite Horizon Program}

In this section we provide a closed form solution for a particular example of the optimal consumption and portfolio problem formulated in section 5 , in which the horizon is infinite. In this analysis, we use a felicity function $u(z, t)=\frac{1}{\alpha} e^{-\delta t} z^{\alpha}$, where $\alpha<1$, and where the discount factor $\delta$ expresses the agent's impatience.

In addition, we assume that the prices of the risky securities follow a geometric Brownian motion given by:

$$
S(t)+\int_{0}^{t} \rho(s) d s=S(0)+\int_{0}^{t} I_{S}(s) \mu d s+\int_{0}^{t} I_{S}(s) \sigma d B(s)
$$


where $I_{S}(t)$ is a diagonal $N \times N$ matrix whose diagonal elements are $S_{n}(t), n=1, \ldots, N$, where $\mu$ is an $N$-dimensional vector of constants, and $\sigma$ is an $N \times M$ matrix of constants. The instantaneous interest rate $r$ is a constant. We assume that $\sigma \sigma^{\top}$ is nonsingular.

Note that since the asset price parameters and the interest rate are constant, the value function $J$ depends only on wealth $W$, the average past consumption $z$, and $t$. Also, it is easy to see that $J(W, z, t)=e^{-\delta t} J(W, z, 0)$, since the felicity function is time separable and with a constant impatience factor. We will therefore focus our attention on the function $J(W, z, 0)$ and henceforth denote this function simply by $J(W, z)$. The differential inequality of (14) becomes

$$
\max \left\{\frac{z^{\alpha}}{\alpha}+\max _{A}\left[\mathcal{D}^{A} J\right]-\delta J, \beta J_{z}-J_{W}\right\}=0
$$

The boundary condition when $W=0$ is

$$
J(0, z)=\frac{1}{\alpha(\alpha \beta+\delta)} z^{\alpha} .
$$

We will show that the optimal solution to the agent's problem takes the form of a ratio barrier policy. The optimal investment decision is to invest constant fractions of wealth in the $N$ risky assets at all times. The optimal consumption is the amount required to keep the ratio of wealth $W$ to average past consumption $z$ less than ${ }^{9}$ a critical number $k^{*}$ in almost all states of nature. If the agent starts at time zero with wealth $W(0)$ and initial consumption experience $z\left(0^{-}\right)$such that $\frac{W(0)}{z\left(0^{-}\right)}$is strictly less than $k^{*}$, then the optimal consumption policy is to consume nothing and wait while $W$ increases on average and $z$ declines until the (random) time $\tau$ when the ratio $\frac{W(\tau)}{z(\tau)}$ is equal to $k^{*}$. Starting from that moment, the agent consumes only when $\frac{W}{z}=k^{*}$ the amount required to keep $\frac{W}{z} \leq k^{*}$ forever in all states of nature.

On the other hand, if the agent starts at time zero with $\frac{W(0)}{z\left(0^{-}\right)}>k^{*}$, then the optimal consumption policy is to take a "gulp" of consumption, reducing $W$ and increasing $z$, to bring the ratio $\frac{W}{z}$ immediately to $k^{*}$. Following this gulp, the optimal amount of consumption is that required to keep $\frac{W}{z} \leq k^{*}$ forever, and consumption occurs only when $\frac{W}{z}=k^{*}$. As we shall see, the erratic behavior of the price of the risky assets implies that the optimal consumption pattern will have "singular" sample paths. In almost all states of nature, the agent consumes a non-trivial amount at infinitely many points of time. However, the times when consumption occurs are a set of Lebesgue measure zero.

In essence, when the wealth in relation to $z$ is too high, the agent will convert wealth into consumption. Conversely, when the wealth relative to $z$ is low, the agent will refrain

\footnotetext{
${ }^{9}$ Recall that we use weak relations and hence $x$ less than $y$ means that $x$ is strictly less than or equal to $y$.
} 
from consumption altogether to accumulate her wealth and in the mean time, will enjoy the satisfaction derived from her past consumption purchases. The singularity of the sample paths of the optimal consumption pattern is caused by the erratic behavior of a Brownian motion. This is a feature of the modeling choice in which the returns on risky securities follow a Brownian motion. The economic content of the solution is the periodicity of consumption, which is in part due to the fact that the agent derives satisfaction from past consumption.

We will employ probabilistic methods to compute the function $J$ and the optimal consumption and portfolio policies. We present the logic behind the optimal solution in two steps. First, we analyze general consumption and portfolio policies of the $k$-ratio barrier form. For any $k>0$, the corresponding $k$-ratio barrier policy is the policy of investing constant fractions of wealth in the $N$ risky assets together with a consumption policy of keeping the ratio $\frac{W}{z}$ less than or equal to $k$ forever in all states of nature and consuming only when $\frac{W}{z}=k$. This policy is followed after an initial "gulp" of consumption if the initial conditions are such that $\frac{w^{\prime}(0)}{z\left(0^{-}\right)}>k$, or after a (random) period of no consumption if $\frac{W(0)}{z\left(0^{-}\right)}<k$. We will show that the expected life-time utility associated with any $k$-ratio barrier policy and initial state $(W, z)$, denoted $J^{k}(I V, z)$, satisfies

$$
\begin{aligned}
\max _{A}\left\{\frac{z^{\alpha}}{\alpha}+\mathcal{D}^{A} J^{k}-\delta J^{k}\right\}=0 & \text { if } W \leq k z \text { and } \\
J_{W}^{k}-\beta J_{z}^{k}=0 & \text { if } \quad W \geq k z
\end{aligned}
$$

Second, we will show that there is a unique value $k^{*}>0$ such that the $k^{*}$-ratio policy together with its associated value function, $J^{*}(W, z)$, satisfy all the conditions of Theorem 2 and hence is the optimal policy.

Before proceeding, we make two observations. First, let $C$ be the optimal consumption pattern with initial state variables $(W, z)$ for a $k$-ratio policy. By the linearity of the dynamics of wealth and the average past consumption, given in (4) and (5), respectively, $a C$ is the consumption pattern for the same $k$-ratio policy with initial state variables $(a W, a z)$ for any scalar $a>0$. Thus $J^{k}(a W, a z)=a^{\alpha} J^{k}(W, z)$. That is, $J^{k}$ is homogeneous of degree $\alpha$ in that $J^{k}(W, z)=z^{\alpha} J^{k}(W / z, 1)$. Second, it is easily seen that the optimal investment strategy $A^{*}$ must be time independent and is a function of $W$ and $z$. Direct computation shows that

$$
A^{*}(W, z)=-\frac{J_{W}^{k}}{W J_{W W}^{k}} \Gamma,
$$

where $\Gamma \equiv\left(\sigma \sigma^{\top}\right)(\mu-r 1)$ and 1 is an $N$-vector of 1's. The investment policy is always propor- 
tional to the vector $\Gamma .^{10}$

The object of control under a $k$-ratio policy is the ratio $\frac{W}{z}$ and the purpose of control is to keep this ratio less than $k$. Suppose that the agent starts from a state $(W, z)$ such that $\frac{W}{z}<k$, and suppose that she invests constant proportions of her wealth in the $N$ risky assets, denoted by $A^{k}$, and consumes nothing until the first time $\tau$ when $\frac{W(\tau)}{z(\tau)}=k$. Also suppose that from $\tau$ on, the agent consumes the minimum amount required to keep the ratio $\frac{W(t)}{z(t)} \leq k$ for all $t>r$. The wealth dynamics of this policy is well-defined by Lions and Sznitman (1984). Let us compute the expected utility $J^{k}(W, z)$ obtained from such a policy.

From the definition of the utility, we can write, for $(W, z)$ such that $\frac{W}{z}<k$ :

$$
\begin{aligned}
J^{k}(W, z) & =\mathrm{E}\left[\int_{0}^{\tau} e^{-\delta s} \frac{z^{\alpha}}{\alpha} e^{-\alpha \beta s} d s+e^{-\delta \tau} J^{k}\left(W(\tau), z e^{-\beta \tau}\right)\right] \\
& =\mathrm{E}\left[\frac{z^{\alpha}}{\alpha(\alpha \beta+\delta)}\left[1-e^{-(\alpha \beta+\delta) \tau}\right]\right]+\mathrm{E}\left[e^{-\delta \tau} J^{k}\left(W(\tau), z e^{-\beta \tau}\right)\right] .
\end{aligned}
$$

Given that $J^{k}$ is homogeneous of degree $\alpha$, for a point on the boundary $(W(\tau), z(\tau)$, $J(W(\tau), z(\tau))=z(\tau)^{\alpha} J(k, 1)$. We thus know that

$$
J^{k}(W(\tau), z(\tau))=a_{k} z(\tau)^{\alpha}
$$

for some constant $a_{k}$. Hence, we can rewrite $J^{k}(W, z)$ as:

$$
J^{k}(W, z)=\frac{z^{\alpha}}{\alpha(\alpha \beta+\delta)}+\left[a_{k}-\frac{1}{\alpha(\alpha \beta+\delta)}\right] z^{\alpha} \mathbf{E}\left[e^{-(\alpha \beta+\delta) \tau}\right] .
$$

Assume that the constant investment policy $A^{k}=b_{k} \Gamma$ for some scalar $b_{k}$. Then starting from a state $\frac{W}{z}<k$, the $\log$ arithm of the ratio $\frac{W}{z}$, before $\frac{W}{z}$ reaching $k$, follows a Brownian motion with drift $\mu_{k}=r+\beta+b_{k} \gamma-b_{k}^{2} \gamma / 2$ and a standard deviation $\sigma_{k}=b_{k} \sqrt{\gamma}$, where $\gamma \equiv[\mu-r 1]^{\top}\left(\sigma \sigma^{\top}\right)^{-1}[\mu-\tau 1]$. From Harrison (1985, proposition 3.23), we conclude that

$$
\mathbf{E}\left[e^{-(\alpha \beta+\delta) \tau}\right]=e^{-\alpha_{k}\left(\log k-\log \frac{W}{z}\right)}=\left(\frac{W}{k z}\right)^{\alpha_{k}}
$$

where

$$
\alpha_{k}=\frac{1}{\sigma_{k}^{2}}\left[\left(\mu_{k}^{2}+2 \sigma_{k}^{2}(\alpha \beta+\delta)\right)^{\frac{1}{2}}-\mu_{k}\right]
$$

Substituting from (36) into (35), we get, when $\frac{W}{z}<k$,

$$
J^{k}(W, z)=\frac{z^{\alpha}}{\alpha(\alpha \beta+\delta)}+\left[a_{k}-\frac{1}{\alpha(\alpha \beta+\delta)}\right] z^{\alpha}\left(\frac{W}{k z}\right)^{\alpha_{k}} .
$$

\footnotetext{
${ }^{10}$ Here we note the two-fund separation property implied by (33); see Merton (1971).
} 
Now, consider the situation when $\frac{w}{z}>k$. The $k$-barrier policy prescribes a jump of size $\Delta=\frac{W-k z}{1+\beta k}$ to a point on the boundary and the corresponding utility is

$$
J^{k}(W, z)=a_{k} \frac{(z+\beta W)^{\alpha}}{(1+\beta k)^{\alpha}}
$$

Notice that (39) implies $J_{W}=\beta J_{z}$ for all $\frac{W}{z}>k$. We then choose the value of the constant $a_{k}$ such that $J_{W}^{k}=\beta J_{z}^{k}$ when $\frac{W}{z}=k$. From this condition and ( 38$)$, we get

$$
a_{k}=\frac{\beta k}{(\alpha \beta+\delta)\left[\alpha_{k}(1+\beta k)-\alpha \beta k\right]}+\frac{1}{\alpha(\alpha \beta+\delta)} \text {. }
$$

To summarize, the value function associated with a constant proportions investment strategy $A^{k}=b_{k} \Gamma$ and a $k$-ratio consumption poljcy is given by

$$
J^{k}(W, z)= \begin{cases}\frac{z^{\alpha}}{\alpha(\delta+\alpha \beta)}+z^{\alpha}\left[\frac{W}{k z}\right]^{\alpha_{k}}\left[a_{k}-\frac{1}{\alpha(\alpha \beta+\delta)}\right] & \text { if } \frac{W}{z} \leq k \\ a_{k}\left(\frac{z+\beta W}{1+\beta k}\right)^{\alpha} & \text { if } \frac{W}{z} \geq k\end{cases}
$$

where $a_{k}$ is given in (40) and $\alpha_{k}$ is given by (37).

Note that in deriving (41) we started by assuming a constant proportions investment strategy $A^{k}=b_{k} \Gamma$. This investment strategy together with the $k$-ratio consumption policy implies the functional form $J^{k}$. If $J^{k}$ were the optimal value function, the optimal investment strategy would be determined through (33). In particular, since the $k$-ratio policy would keep $\frac{W}{z} \leq k$, the optimal investment strategy would be equal to $\Gamma /\left(1-\alpha_{k}\right)$. Thus, if there exists a solution to the system of equations composed of (37) and $b_{k}=1 /\left(1-\alpha_{k}\right)$ and this solution is less than 1 to make $J^{k}$ a concave function, then we have produced a consistent $J^{k}$ in that the implied investment behavior of $J^{k}$ generates the functional form of $J^{k}$. We need to make enough assumptions on the parameters of the problem to ensure that a desired solution of $\alpha_{k}$ exist. We assume throughout this section that:

Assumption 1 The parameters of the problem satisfy

$$
\delta>\alpha r+\frac{\alpha \gamma}{2(1-\alpha)} \text { and }(1-\alpha) \beta>\delta-r
$$

Given these restrictions, we can easily establish the following result, whose proof uses elementary algebra and is left for the reader. 
Lemma 1 There exist a unique $\alpha_{k}<1$ that solves the system of equations of (37) and $b_{k}=$ $1 /\left(1-\alpha_{k}\right)$ and

$$
\alpha_{k}=\frac{[\gamma / 2+(r+\beta)+(\alpha \beta+\delta)]-\sqrt{[\gamma / 2+(r+\beta)+(\alpha \beta+\delta)]^{2}-4(r+\beta)(\alpha \beta+\delta)}}{2(r+\beta)} .
$$

Moreover, $\alpha_{k}>\alpha$ and $\alpha_{k}$ is independent of $k$.

As a consequence of $\alpha_{k}$ being independent of $k, A^{k}=\Gamma /\left(1-\alpha_{k}\right)$ is also independent of $k$. We will henceforth denote by $\alpha^{*}$ the solution characterized in Lemma 1 and by $A^{*}$ the constant proportions investment strategy $\Gamma /\left(1-\alpha^{*}\right)$. It is also understood that the $\alpha_{k}$ is replaced by $\alpha^{*}$ in the definition of $a_{k}$ of (40). The following proposition gives properties of $J^{k}$ defined in (41).

Proposition 2 The function $J^{k}$ of (41) with $\alpha_{k}$ replaced by $\alpha^{*}$ is increasing, continuous, strictly concave, and twice differentiable, has continuous first derivatives, has continuous second derivatives except possibly on the smooth manifold $\mathcal{M}_{k} \equiv\{(W, z): W=k z\}$, and satisfies, together with $A^{*}$,

$$
\begin{aligned}
\frac{z^{\alpha}}{\alpha}+\mathcal{D}^{A^{*}} J^{k}-\delta J^{k}=0 & \text { if } \quad W \leq k z \quad \text { and } \\
J_{W}^{k}-\beta J_{z}^{k}=0 & \text { if } \quad W \geq k z
\end{aligned}
$$

and the boundary conditions

$$
\begin{gathered}
J(0, z)=\frac{1}{\alpha(\alpha \beta+\delta)} z^{\alpha} \text { and } \\
\lim _{t \rightarrow \infty} \mathrm{E}\left[e^{-\delta t} J^{k}\left(W_{k}(t), z_{k}(t)\right)\right]=0,
\end{gathered}
$$

where $W_{k}$ and $z_{k}$ are the wealth and the average past consumption associated with the $k$-ratio consumption policy and the investment strategy $A^{*}$. In addition, $e^{-\delta t} J^{k}$ satisfies the growth condition of (25).

Proof. All the assertions except for the last can be verified by direct computations using the fact that $\alpha<\alpha^{*}<1$. For the last boundary condition note that $W_{k}(t) \leq k z_{k}(t)$ for all $t>0$, and hence $J^{k}\left(W_{k}(t), z_{k}(t)\right)$ is less than $a_{k} z_{k}^{\alpha}(t)$, where we recall that $a_{k}$ is defined in (40). Note that

$$
\begin{aligned}
z_{k}(t) & =z\left(0^{-}\right) e^{-\beta t}+\beta \int_{0}^{t} e^{-\beta(t-s)} d C_{k}(s) \\
& <z\left(0^{-}\right) e^{-\beta t}+\left[\beta C_{k}(t)\right],
\end{aligned}
$$


where $C_{k}$ denotes the $k$-ratio consumption policy. From the budget constraint of (4), we can see that $C_{k}(t) \leq \hat{W}(t)$ a.s., where $\hat{W}(t)$ is the wealth realized at time $t$ when following the portfolio rule $A^{*}$ with no consumption withdrawal before $t$. Using the dynamics of $\hat{W}$, one can easily verify that

$$
\lim _{t\rceil \infty} \mathrm{E}\left[e^{-\delta t} J\left(W_{k}(t), z_{k}(t)\right)\right] \leq \lim _{t\rceil \infty} \mathrm{E}\left[a_{k} e^{-\delta t} z_{k}^{\alpha}(t)\right] \leq \lim _{t\rceil \infty} \mathrm{E}\left[a_{k} e^{-\delta t}\left[z\left(0^{-}\right) e^{-\beta t}+\beta \hat{W}(t)\right]^{\alpha}\right]
$$

But

$$
\left[z(0) e^{-\beta t}+\beta \hat{W}(t)\right]^{\alpha} \rightarrow[\beta \hat{W}(t)]^{\alpha} \text { uniformly in } \omega \text { as } t \uparrow \infty
$$

hence

$$
\begin{aligned}
\lim _{t \uparrow \infty} \mathrm{E}\left[e^{-\delta t}\left(z(0) e^{-\beta t}+\beta \hat{W}(t)\right)^{\alpha}\right] & =\lim _{t \uparrow \infty} \mathrm{E}\left[e^{-\delta t} \beta^{\alpha} \hat{W}^{\alpha}(T)\right] \text { and thus } \\
\lim _{t \uparrow \infty} \mathrm{E}\left[e^{-\delta t} J\left(W_{k}(t), z_{k}(t)\right)\right] \leq & \lim _{t \uparrow \infty} \mathrm{E}\left[e^{-\delta t} \beta^{\alpha} \hat{W}^{\alpha}(t)\right] .
\end{aligned}
$$

But

$$
\begin{aligned}
& \mathbf{E}[\hat{W}(t)]^{\alpha}=W(0) e^{\left\{\alpha\left[r+\frac{\gamma\left(1-2 a^{*}\right)}{2\left(1-a^{*}\right)^{2}}\right]+\frac{a^{2} \gamma}{2\left(1-a^{*}\right)^{2}}\right\} t} \\
& <W(0) e^{\left\{\alpha^{\cdot}\left[r+\frac{\gamma\left(1-2 a^{*}\right)}{2\left(1-a^{*}\right)^{2}}\right]+\frac{a^{* 2} \gamma}{2\left(1-\alpha^{*}\right)^{2}}\right\} t} \\
& \leq W(0) e^{\left[\alpha^{\bullet}(r+\beta)+\frac{a^{\bullet} \gamma}{2\left(1-\alpha^{*}\right)}\right] t-\alpha^{\bullet} \beta t} \\
& \leq W(0) e^{(\alpha \beta+\delta) t-\alpha \cdot \beta t},
\end{aligned}
$$

where the first inequality follows from the property that $\alpha<\alpha^{\circ}$, and the last inequality from the definition of $\alpha^{*}$. Hence $\mathbf{E}\left[e^{-\delta t} \hat{W}^{\alpha}(t)\right] \leq W(0) e^{\beta\left(\alpha-\alpha^{*}\right) t}$. Noting that $\alpha-\alpha^{*}<0$, the required result follows.

The last assertion is obvious.

Now, we show that a $k$-ratio policy financed by $A^{*}$ is an admissible policy in (24). Moreover, the function $J^{k}$ is indeed the expected utility that the agent gets from following this $k$-ratio policy.

Proposition 3 The wealth process and the consumption pattern associated with a $k$-ratio barrier consumption policy financed by the investment strategy $A^{*}$ satisfy (23). In addition, the function $e^{-\delta t} J^{k}(W, z)$ gives the expected utility of following these policies at time $t$. 
Proof. Let $W_{k}, C_{k}$, and $z_{k}$ denote the wealth, the consumption, and the average past consumption associated with the $k$-ratio consumption policy financed by $A^{*}$. Recall from the proof of Proposition 2 that $C_{k}(t) \leq \hat{W}(t)$ a.s., where we recall that $\hat{W}(t)$ is the wealth realized at time $t$ when following the investment strategy $A^{*}$ with no consumption withdrawal before $t$. Similarly, $W_{k}(t) \leq \mathfrak{V}(t)$ a.s. It is easily verified that $\hat{W}$ satisfies (23) since it is a geometric Brownian motion and thus $\hat{W}(t)$ is log-normally distributed. The first assertion follows.

For the second assertion, identical arguments used to prove Theorem 2 show that

$$
\mathrm{E}\left[\int_{t}^{s} e^{-\delta v} \frac{z_{k}(v)^{\alpha}}{\alpha} d v+e^{-\delta s} J^{k}\left(W_{k}(s), z_{k}(s)\right)\right]=e^{-\delta t} J^{k}\left(W_{k}(t), z_{k}(t)\right)
$$

where we have used Proposition 2. The assertion follows from monotone convergence theorem by letting $s \rightarrow \infty$.

From the above discussions, we know that any $k$-ratio consumption policy together with $A^{*}$ satisfies many of the sufficient conditions for optimality. All policies, except one, however, fail to satisfy the differential inequality (31). The unique barrier policy that satisfies inequality (31) is the optimal solution, which is recorded in the following proposition.

Proposition 4 Let

$$
k^{*}=\frac{1-\alpha^{*}}{\beta\left(\alpha^{*}-\alpha\right)} .
$$

Then $k^{*}>0$ and the solution to (24) consists of the investment strategy $A^{*}$ and the $k^{*}$-ratio barrier consumption policy.

Proof. First $k^{*}>0$ by lemma 1.

Next let the value function associated with the $k^{*}$-ratio barrier policy be $J^{*}$. In view of Proposition 2, we only need to show that $J^{*}$ satisfies:

$$
\begin{aligned}
J_{W}^{*}-\beta J_{z}^{*} \leq 0 & \text { if } W \leq k^{*} z \text { and } \\
\frac{z^{\alpha}}{\alpha}+\mathcal{D}^{A^{*}} J^{*}-\delta J^{*} \leq 0 & \text { if } W \geq k^{*} z
\end{aligned}
$$

We start with the first inequality. Using the definition of $J^{*}$, we can write $J_{W}^{*}-\beta J_{z}^{*}$ in the region $W \leq k^{*} z$ as:

$$
\begin{aligned}
J_{\text {W }}^{*}-\beta J_{z}^{*} & =a z^{\alpha-1} g\left(\frac{k^{*} z}{W}\right) \text { where } \\
g(y) & =\beta\left(\alpha^{*}-\alpha\right) y^{\alpha^{*}}+\frac{\alpha^{*}}{k^{*}} y^{\left(\alpha^{*}-1\right)}-\frac{\beta}{\alpha \beta+\delta}
\end{aligned}
$$


where $a$ is a constant. Note that $g(1)=0$, and that since $\alpha^{*}>0, g(y) \uparrow \infty$ as $y \uparrow \infty$.

Computing the derivative of $g$, we get

$$
\frac{d g}{d y}=\alpha^{*} \beta\left(\alpha^{*}-\alpha\right) y^{\alpha^{*}-1}+\frac{\alpha^{*}\left(\alpha^{*}-1\right)}{k^{*}} y^{\alpha^{*}-2} .
$$

Substituting $k^{*}$ in the expression for $\frac{d g}{d y}$, we conclude that $\frac{d g}{d y}=0$ for $y=1$, and that $\frac{d g}{d y}>0$ for $y>1$. It then follows that $J_{W}^{*}-\beta J_{z}^{*} \geq 0$, for all points $(W, z)$ such that $W \leq k^{*} z$.

Now, we verify the second inequality. Consider any point $\underline{x} \equiv(W, z)$ such that $W \geq k^{*} z$. Let $\underline{a}$ be the point on the intersection of the line $W=k^{*} z$ and the straight line passing through the point $\underline{x}$ with slope $\frac{d W}{d z}=-\frac{1}{\beta}$. In other words, $\underline{a}$ is the point on the boundary $W=k^{*} z$, to which one would jump if one starts at $\underline{x}$. By construction, $J^{*}(\underline{x})=J^{*}(\underline{a})$.

Let

$$
f(W, z)=\frac{z^{\alpha}}{\alpha}+J_{W}^{*} r W-\frac{J_{W}^{* 2}}{2 J_{W W}^{*}} \gamma-J_{z}^{*} \beta z,
$$

and note that $f(\underline{a})-\delta J^{*}(\underline{a})=0$. Applying the fundamental theorem of calculus along the straight line connecting $\underline{a}$ and $\underline{x}$, we obtain

$$
f(\underline{x})-f(\underline{a})=\int_{\underline{a}}^{\underline{x}} f_{W} d W+f_{z} d z .
$$

Noting that along the line connecting $\underline{a}$ and $\underline{x}$, we have $d z=-\beta d W$, and that $d W>0$ in the direction from $\underline{a}$ to $\underline{x}$, it then follows that $f_{W}-\beta f_{z} \leq 0$ is sufficient to conclude that

$$
\frac{z^{\alpha}}{\alpha}+J_{W}^{*} r W-\frac{J_{W}^{* 2}}{2 J_{W W}^{*}} \gamma-J_{z}^{*} \beta z-\delta J^{*} \leq 0 \quad \text { if } \quad \frac{W}{z} \geq k^{*} .
$$

Computing $f_{W}-\beta f_{z}$ in the region $W \geq k^{*} z$, and using the properties of $\alpha^{*}$, the reader can easily verify that $f_{W}-\beta f_{z} \leq 0$ for $k^{*}=\frac{1-\alpha^{*}}{\beta\left(\alpha^{*}-\alpha\right)}$.

The following proposition records the optimal consumption and investment policy starting at $t=0$. The optimal policy from any $t>0$ can be constructed similarly.

\section{Proposition 5 Let}

$$
A^{*}(t)=\frac{1}{1-\alpha^{*}} \Gamma \text { for all } t
$$

and let the budget feasible consumption process $C^{*}$, which has continuous sample paths almost surely, be given by:

$$
C^{*}(t)=\Delta C^{*}(0)+\int_{0}^{t} \frac{W^{*}(s)}{1+\beta k^{*}} d l(s) \quad P-a . s .
$$


where

$$
\begin{aligned}
\Delta C^{*}(0) & =\max \left\{0, \frac{W(0)-k^{*} z\left(0^{-}\right)}{1+\beta k^{*}}\right\}, \\
l(t) & =\sup _{0 \leq s \leq t}\left[\log \frac{\hat{W}(s)}{\hat{z}(s)}-\log k^{*}\right]^{+} P-a . s_{.}, \\
\hat{W}(t) & =\left(W_{0}-\Delta C^{*}(0)\right) e^{\left(\left[r+\frac{\gamma\left(1-2 a^{*}\right)}{2\left(1-\alpha^{*}\right)^{2}}\right] t+\left[\frac{1}{\left(1-\alpha^{*}\right)} \Gamma^{\top} \sigma\right] B(t)\right)} \quad P-\text { a.s., } \\
\hat{z}(t) & =\left(z_{0}+\beta \Delta C^{*}(0)\right) e^{-\beta t} \quad P-\text { a.s. },
\end{aligned}
$$

and where $W^{*}(s)$ and $z^{*}(s)$ are the state variables associated with $C^{*}$. The strategy $A^{*}$ and $C^{*}$ defined above are the unique optimal solution for the agent's problem.

Proof. To prove this proposition, all we need to show is that the above strategy is the ratio barrier strategy associated with $k^{*}$. The $k^{*}$ ratio barrier policy calls for a jump at $t=0$, if $\frac{W(0)}{z\left(0^{-}\right)}>k^{*}$. The size of the jump $\Delta$ is calculated to achieve the condition $\frac{W(0)-\Delta}{z\left(0^{-}\right)+\beta \Delta}=k^{*}$, from which we compute $\Delta$ as in (47).

Let us proceed now to compute the consumption process after $t=0$. The $k^{*}$-ratio barrier policy is equivalent to the condition that

$$
\begin{aligned}
\frac{W^{*}(t)}{z^{*}(t)} & \leq k^{*} \forall t, P-a . s . \quad \text { or } \\
\log \frac{W^{*}(t)}{z^{*}(t)} & \leq \log k^{*} \quad \forall t, P-a . s .
\end{aligned}
$$

Now consider the "unregulated" process $(\hat{W}, \hat{z})$, which gives the wealth of the agent and her past average consumption under the assumption that no consumption takes place after the initial jump at $t=0$. The values of $\hat{W}(t)$ and $\hat{z}(t)$ are given in (49) and (50). The ratio $\frac{\hat{W}^{\prime}(t)}{\dot{z}(t)}$ will fail to satisfy condition (51) for some periods and for some sample paths, and the idea of the solution is to "regulate" this ratio using the consumption process $C^{*}$ to ensure that at the optimal solution condition (51) is satisfied.

To achieve this regulation, define the process

$$
l(t)=\sup _{0 \leq s \leq t}\left[\log \frac{\hat{W}^{\prime}(s)}{\hat{z}(s)}-\log k^{*}\right]^{+} P-a . s .
$$

and let the "regulated" process $\log \frac{W^{*}}{z^{*}}$ be given by:

$$
\log \frac{W^{*}(t)}{z^{*}(t)}=\log \frac{\hat{W}(t)}{\hat{z}(t)}-l(t) \quad P-a . s .
$$

For each state $\omega$, the sample path $l(\omega,$.$) has the following properties:$ 
- $l(\omega,$.$) is increasing and continuous with l(\omega, 0)=0$, for almost all $\omega$.

- $\log \frac{W^{*}(t)}{z^{*}(t)} \leq \log k^{*}$ for all $t \geq 0$, P-a.s.

- $l(\omega,$.$) increases only when \log \frac{W^{*}(\omega, t)}{z^{*}(\omega, t)}=\log k^{*}$.

This "regulated" process $\left(\log \frac{W^{\bullet}(t)}{z^{*}(t)}\right)$ is a candidate for the logarithm of the ratio of the state variables associated with the optimal consumption plan, since from the above construction, we can easily conclude that condition (51) is satisfied. The question now becomes whether there exists a feasible consumption process $C^{*}$ that could enforce the relationship in (53). Expanding both sides of (53) using Itô's lemma, we get

$$
\begin{aligned}
\log \frac{W^{*}(t)}{z^{*}(t)}= & \log \frac{W(0)-\Delta C^{*}(0)}{z\left(0^{-}\right)+\beta \Delta C^{*}(0)}+\int_{0}^{t}\left[r+\beta+\frac{\gamma\left(1-2 \alpha^{*}\right)}{2\left(1-\alpha^{*}\right)^{2}}\right] d s \\
& +\int_{0}^{t} \frac{1}{\left(1-\alpha^{*}\right)} \Gamma^{\top} \sigma d B(s)-\int_{0}^{t}\left[\frac{1}{W^{*}(s)}+\frac{\beta}{z^{*}(s)}\right] d C^{*}(s) \quad P-\text { a.s. } \\
\log \frac{\hat{W}(t)}{\hat{z}(t)}= & \log \frac{W(0)-\Delta C^{*}(0)}{z\left(0^{-}\right)+\beta \Delta C^{*}(0)}+\int_{0}^{t}\left[r+\beta+\frac{\gamma\left(1-2 \alpha^{*}\right)}{2\left(1-\alpha^{*}\right)^{2}}\right] d s \\
& +\int_{0}^{t} \frac{1}{\left(1-\alpha^{*}\right)} \Gamma^{\top} \sigma d B(s) \quad P-a . s .
\end{aligned}
$$

Thus, we conclude that the consumption process given by:

$$
\int_{0}^{t}\left[\frac{1}{W^{*}(s)}+\frac{\beta}{z^{*}(s)}\right] d C^{*}(s)=l(t) \quad P-a . s .
$$

satisfies the condition in (53). Note from the above equation that $C^{*}$ increases only when $l$ increases for all sample paths. Therefore, $C^{*}$ increases only when $\frac{W^{*}(t)}{z^{*}(t)}=k^{*}$ and hence condition (27) of Theorem 2 is satisfied.

Now let the process $y$ be given by $y(t)=\frac{W^{\bullet}(t) z^{\bullet}(t)}{\beta W^{\bullet}(t)+z^{\bullet}(t)}$. From Itô's lemma, we get:

$$
y(t) l(t)=y(0) l(0)+\int_{0}^{t} y(s) d l(s)+\int_{0}^{t} l(s) d y(s) \quad P-a . s .
$$

Noting that $l(0)=0, P-a . s .$, and that $d l(s)=\frac{d C^{\bullet}(s)}{y(s)}$, we find that

$$
y(t) l(t)=C^{*}(t)+\int_{0}^{t} l(s) d y(s) \quad P-a . s .
$$

Integrating the second term in the right-hand side by parts, we have that

$$
C^{*}(t)=\int_{0}^{t} \frac{W^{*}(s) z^{*}(s)}{\beta W^{*}(s)+z^{*}(s)} d l(s) \quad P-a . s .
$$


But from the properties of $l$, we know that $l$ increases only when $\frac{W^{*}(s)}{z^{*}(s)}=k^{*}$. We thus conclude that

$$
C^{*}(t)=\int_{0}^{t} \frac{W^{*}(s)}{1+\beta k^{*}} d l(s) \quad P-a . s .
$$

The solution we constructed has the following features. After the initial (possible) gulp, the agent consumes the minimum possible amount required to keep the marginal value of wealth greater than ( $\beta$ times) the marginal value of $z$. Consumption takes place only when these marginal values are equalized. If the marginal value of $z$ drops below ( $\beta$ times) the marginal value of wealth, the agent stops consuming. These rules result in a consumption process with singular sample paths. Consumption occurs at uncountably infinite number of times, but the set of all times when consumption occurs has Lebesgue measure zero, for almost all sample paths. This result occurs because of the unbounded variation property of the Brownian motion sample paths.

\section{Comparative Statics}

It is interesting to compare the behavior described in the previous section to the behavior of an agent with a time-additive utility function defined on the subspace of absolutely continuous consumption patterns and given by:

$$
\mathrm{E}\left[\int_{0}^{\infty} e^{-\delta t} \frac{c(t)^{\alpha}}{\alpha} d t\right]
$$

where $c(t)$ is the consumption rate at time $t$. Note that the utility function in (55) is the limit of the utility function analyzed in section 6 as $\beta \uparrow \infty$.

Consider the consumption-investment problem of an agent whose preferences are given by (55) and who faces the investment opportunities given by (30) and a constant riskless rate. Merton (1971) shows that the agent's optimal policy is to consume continuously at a rate equal to a constant fraction of total wealth, and to invest constant proportions $\frac{1}{1-\alpha} \Gamma$ of his wealth in the risky assets.

Now compare the investment policies of the agent we studied in this paper with those of Merton's time-additive agent, assuming that the corresponding felicity functions have the same curvature $\alpha$, and that the agents have the same time preference parameter $\delta$, and face the same investment opportunities. From lemma 1, we know that $\alpha^{*}>\alpha$. Furthermore, $\lim _{\beta i \infty} \alpha^{*}=\alpha$. Hence the agent whose preferences exhibit local intertemporal substitution invests more in the 
risky assets and appears to be less risk averse. Furthermore, the weaker the effect of local substitution (higher $\beta$ ), the less that the agent invests in risky assets, ceteris paribus. One could explain such a behavior by arguing that the fact that past consumption affects current and future utility leads the agent to tolerate higher probabilities of reduced future level of wealth and hence take more risky positions.

The reader can easily verify that $\frac{\partial A^{*}}{\partial r}<0$, and $\frac{\partial A^{*}}{\partial \delta}<0$. In other words, higher values of riskless rate and higher discount factors lead to less investment in the risky assets, ceteris paribus.

Another interesting property of the solution for the non-time-additive utility is the steady state distribution and the expected value of the the ratio $\frac{W^{*}}{z^{\circ}}$. Following the computations in Harrison $(1985, \S 5.6)$, we conclude that, from any starting point $\left(W_{0}, z_{0}\right)$, as $t \rightarrow \infty$ :

$$
P_{0}\left\{\frac{W(t)}{z(t)} \leq x\right\} \rightarrow \begin{cases}\left(\frac{x}{k^{*}}\right)^{\frac{2 \mu^{*}}{\sigma^{*}}} & \text { if } x \leq k^{*} \\ 1 & \text { if } x \geq k^{*}\end{cases}
$$

and

$$
\mathrm{E}_{0}\left[\frac{W(t)}{z(t)}\right] \rightarrow \frac{\mu^{*}}{\mu^{*}+\sigma^{* 2} / 2} k^{*},
$$

where $P_{0}$ and $\mathrm{E}_{0}$ are the probability distribution and expectation, respectively, conditional on starting at $(W(0), z(0))$ and where

$$
\begin{aligned}
\mu^{*} & =r+\beta+\frac{\gamma}{1-\alpha^{*}}-\frac{\gamma}{2\left(1-\alpha^{*}\right)^{2}} \\
\sigma^{*} & =\frac{\sqrt{\gamma}}{\left(1-\alpha^{*}\right)}
\end{aligned}
$$

and where $\gamma \equiv[\mu-r 1]^{\top}\left(\sigma \sigma^{\top}\right)^{-1}[\mu-r 1]$.

Denote $\lim _{t \rightarrow \infty} \mathbf{E}_{0}\left[\frac{W(t)}{z(t)}\right]$ by $\left[\frac{W}{z}\right]_{\infty}$. The reader ca $n$ easily verify that:

$$
\frac{\partial}{\partial \beta}\left[\frac{W}{z}\right]_{\infty}>0, \quad \frac{\partial}{\partial \delta}\left[\frac{W}{z}\right]_{\infty}>0, \quad \frac{\partial}{\partial r}\left[\frac{W}{z}\right]_{\infty}>0, \text { and } \quad \frac{\partial}{\partial \sigma}\left[\frac{W}{z}\right]_{\infty}<0 .
$$

One can intuitively understand these relationships as follows. The ratio of wealth to average past consumption eventually settles down to $\left[\frac{W}{z}\right]_{\infty}$. Wealth is the investment in risky assets which the agent holds for purposes of future consumption, whereas $z$ is an indication of his past consumption experience, which also contributes to his future satisfaction. The agent decides on an optimal division between these two sources of future satisfaction. This division is naturally dependent on the strength of the local substitution effect and the outside investment 
opportunities. When the local substitution effect is high (low $\beta$ ), the agent tends to derive relatively more satisfaction from $z$, and hence $\left[\frac{W}{z}\right]_{\infty}$ is low. On the other hand, when the investment opportunities are more attractive (high $r$ or low $\sigma$ ), $\left[\frac{W}{z}\right]_{\infty}$ is high, reflecting more emphasis on deriving future satisfaction from wealth.

\section{Equilibrium Risk Premium}

In this section, we briefly state some of the implications of the form of utility studied in section 6 on asset prices in equilibrium. We adopt the representative agent equilibrium framework of Cox, Ingersoll and Ross (1985a). In the general framework of section 4, we note that the optimal investment policy implies the mutual fund separation results of Breeden (1979) and Merton (1971). Thus the Intertemporal Capital Asset Pricing Model of Merton (1973) holds in equilibrium. In the special case of constant parameter production processes, the constant proportion optimal policy given in (33) implies two-fund separation. Therefore, the Capital Asset Pricing Model holds. However, the Consumption Capital Asset Pricing Model of Breeden (1979) fails to hold because the marginal utility of instantaneous consumption is no longer equal to the marginal utility of wealth. For similar results, see Bergman (1985) and Grossman and Laroque (1990).

Next, we consider the determination of the risk premium. Suppose, for simplicity ${ }^{11}$, that there is one risky production technology whose rates of return follow a $(\mu, \sigma)$-Brownian with $\mu$ and $\sigma$ being strictly positive scalar such that $\alpha \mu \leq \delta .{ }^{12}$ Consider an agent with preferences as given in section 6. Using arguments similar to those of Cox, Ingersoll and Ross (1985b), and using the computations of Harrison $(1985, \S 3.2)$, we conclude that the equilibrium riskless rate, $r$, is given by:

$$
\begin{aligned}
r & =\mu-(1-\bar{\alpha}) \sigma^{2} \text { where } \\
\bar{\alpha} & =\frac{1}{\sigma^{2}}\left[\sqrt{\left(\mu+\beta-\sigma^{2} / 2\right)^{2}+2 \sigma^{2}(\alpha \beta+\delta)}-\left(\mu+\beta-\sigma^{2} / 2\right)\right] .
\end{aligned}
$$

In contrast, if the agent has a time additive utility function whose felicity has the same curvature $\alpha$ and the same time discount parameter $\delta$, the interest rate, is given by $\mu-(1-\alpha) \sigma^{2}$. Given that $\alpha \mu \leq \delta$, the reader can easily verify that $\bar{\alpha}>\alpha$ and hence the risk premium, which

\footnotetext{
${ }^{11}$ This result can be easily generalized to the case of many production technologies.

${ }^{12}$ More specifically, $V(t)$, the level of risky capital at time $t$ starting from one unit continuously reinvested, evolves according to the following equation: $d V(t)=\mu V(t) d t+\sigma V(t) d B(t)$, where $B$ is a one-dimensional standard Brownian motion. Note that we use $\mu$ and $\sigma$ in a different meaning from that in section 6 , and $\delta$ is the parameter of time discount.
} 
is the excess of $\mu$ over the riskless rate, is lower in the case of preferences with local substitution. This is consistent with our earlier observations that the agent whose preferences exhibit local substitution behaves in a less risk averse manner compared to an agent with time-additive utility. In addition, as local substitution decreases ( $\beta$ increases), the risk premium increases.

\section{Temporal Aggregation and Properties of Consumption Series}

Purchases of durable goods are typically measured periodically. Recorded purchases are thus a time aggregate of purchases during the measurement period. It is useful to examine the properties of the process of measured purchases as predicted by the model developed in this paper.

The properties of recorded purchases for an individual will depend mainly on two factors: the wealth relative to the stock of the durable good, or the ratio $\frac{W}{z}$, at the beginning of the measurement period and the length of the measurement period relative to the half-life of the durable good. For example, if one measures the daily purchases of a durable good with a long half-life for an individual who starts with a ratio $\frac{W}{z}$ much lower than the critical ratio $k^{*}$, then one will record zero purchases with very high probability. On the other extreme, if one measures the quarterly purchases of a durable good with a short half-life for an individual who starts with a ratio $\frac{W}{z}$ very close to the boundary value $k^{*}$, then one will observe, with high probability, "smooth" consumption over successive quarters.

The interplay between the length of the measurement period, relative to the half-life of the durable good, and the initial value of $\frac{W}{z}$ can best be illustrated by simulating the process of purchases of the durable good for a typical individual. We assume an economy with two investment opportunities: a riskless asset with constant interest rate $r=6 \%$, and a risky asset whose price follows a geometric Brownian motion process with instantaneous expected rate of return $\mu=14.8 \%$, and instantaneous standard deviation of the rate of return $\sigma=22 \%$. The individual's felicity function is given by ${ }^{13} u(x, t)=\frac{-1}{1.235} e^{-0.06 t} x^{-1.235}$.

We simulate the daily behavior of an individual who follows the optimal solution prescribed in section 6 . We than aggregate his purchases of the durable good over the period of measurement, which we take to be one week, one month or one quarter. We report the autocorrelation of changes in the purchases in two successive measurement periods. To study the dependency

\footnotetext{
${ }^{13}$ The astute reader will observe that this utility function does not satisfy the nonnegativity condition we used in proving the verification theorem. We note that this utility function gives rise to a well posed optimization problem having an optimal solution.
} 
of this statistic on the initial value, we start the measurement from the twenty different states that correspond to the twenty values $\{1.00,0.95,0.90, \cdots, 0.05\}$ on the steady-state distribution function reported in (56). Finally, we report these statistics for durable goods of half-life one week, one month and two years. We use 1000 data points to estimate each statistic and hence the standard error on all reported autocorrelations is 0.0316 .

Figures 1 to 3 report the autocorrelation of changes in the purchases of a durable good with half-life one week, one month and two years, respectively. Each figure reports the autocorrelations for weekly, monthly and quarterly measurements. Note that all measured autocorrelations are negative. This reflects the periodic nature of purchases of durables. Above-average changes of purchases are more likely to be followed by below-average changes. We also observe a clear pattern in the value of the autocorrelations. First, the closer the starting state to the critical boundary is, the lower, in absolute value, is the autocorrelation. Second, the longer the observation period is, the lower is the absolute value of the autocorrelation. This is a reflection of the effect of time aggregation. As more of the purchases are lumped together in one number, the variability of recorded changes in purchases within periods of measurement is reduced.

Figure 4 reports the autocorrelation of changes in the quarterly purchases of the durable good. The figure displays the autocorrelations for durable goods with half-life one week, one month and two years. The figure shows that the shorter the half-life of the good is, the smaller is the absolute value of the autocorrelations. This reflects the fact that the optimal policy for short-lived durables is to purchase small quantities frequently in contrast with the optimal policy for long-lived durables which calls for sporadic and large purchases.

The autocorrelation of changes in the purchases of the durable differ, in statistically significant way, across starting states. The following table reports the average, across initial positions, of autocorrelations of simulated changes in purchases for different durable goods and different measurement periods.

\begin{tabular}{|l|c|c|c|}
\hline \multirow{2}{*}{$\begin{array}{l}\text { Half } \\
\text { Life }\end{array}$} & \multicolumn{3}{|c|}{ Measurement Period } \\
\cline { 2 - 4 } & Weekly & Monthly & quarterly \\
\hline 1 week & -0.788 & -0.702 & -0.541 \\
1 month & -0.758 & -0.700 & -0.614 \\
2 years & -0.751 & -0.712 & -0.731 \\
\hline
\end{tabular}

Observe that if the process of changes in purchases were ergodic, then the reported average cross-sectional autocorrelation would be, asymptotically, equal to the autocorrelation from one sample path of the process. Although, we are unable to prove ergodicity of the changes in 
purchases, we would like to illustrate the "typical" sample path behavior of purchases over some long horizon. Figures 5 and $\mathbf{6}$ show simulated sample paths of quarterly purchases of durable goods with half-life one month and two years, respectively. In addition, both figures show the sample path of the agent's wealth. The contrast between figure $\mathbf{5}$ and $\mathbf{6}$ illustrates clearly the effects of durability on the variability of changes in purchases relative to changes in wealth. A durable good with a longer half-life will be purchased less frequently and in bigger amounts, relative to wealth, than a durable good with a shorter half-life. As a result, purchases of a durable good with short half-life will be "smoother" than purchases of a good with long half-life.

Finally, we remind the reader that all of these results apply only to the purchases of an individual. More work needs to be done to derive the aggregate purchases. We refer the reader to Caballero (1991), and the references therein, for more on this important topic.

\section{Concluding Remarks}

In this paper, we heuristically discussed necessary conditions and provided sufficient conditions for a consumption and portfolio policy to be optimal for a class of time-nonseparable preferences that consider consumptions at nearby dates to be almost perfect substitutes. Our model also admits an alternative interpretation as a model of irreversible purchases of a durable good whose service flow deteriorates as the stock of the good ages. We demonstrated our general theory by explicitly solving in closed form the optimal consumption and portfolio policy for a particular felicity function when the prices of the risky assets follow a geometric Brownian motion process.

We have explicitly solved for the equilibrium interest rate in a Cox, Ingersoll, Ross (1985) type economy with a physical production technology whose rate of return follows a Brownian motion. In addition, we provided some simulation results that demonstrate the properties of the consumption/ purchase series for durable goods with different half-lives.

Two avenues of future research deserve attention. First, we can incorporate the notion of habit formation into our model while preserving the feature that consumptions at nearby dates are almost perfect substitutes. This can be accomplished by defining two weighted averages of past consumption, $z_{1}$ and $z_{2}$, where $z_{1}$ has heavier weights on more recent past consumption than $z_{2}$ and $z_{2}$ has heavier weights on more distant past consumption than $z_{1}$. Consider felicity functions defined on $z_{1}$ and $z_{2}$, denoted by $u\left(z_{1}, z_{2}, t\right)$, with the property that $u_{12}>0$, where 
$u_{12}$ denotes the cross partial derivative of the first two arguments of $u$. In this formulation, the higher the distant past consumption is, the higher the marginal felicity for an additional unit of more recent past consumption will be. Therefore, we can interpret $z_{2}$ as a living standard. This formulation treats consumption at nearby dates as close substitutes and incorporates the notion that "habits" are formed not over a short period of time but rather over longer horizons.

Second, the interest rate derived in Section 8 is of limited interest since it implies a flat yield curve. One needs to explore possible specifications of the production technology to derive explicit and interesting term structure of interest rates.

\section{References}

1. K. Arrow and M. Kurz, Public Investment, the Rate of Return, and Optimal Fiscal Policy, Johns Hopkins Press, Baltimore, 1970.

2. Y. Bergman, Time Preference and Capital Asset Pricing Model, Journal of Financial Economics 14, 145-160, 1985.

3. D. Breeden, An Intertemporal Asset Pricing Model with Stochastic Consumption and Investment Opportunities, Journal of Financial Economics 7, pp. 265-296, 1979.

4. R. Caballero, Durable Goods: An Explanation for Their Slow Adjustment, mimeo, Columbia University, 1991.

5. G. Constantinides, Habit Formation: A Resolution of the Equity Premium Puzzle, Journal of Political Economy 98, pp. 519-543, 1990.

6. J. Cox and C. Huang, Optimal Consumption and Portfolio Policies when Asset Prices follow a Diffusion Process, Journal of Economic Theory 49, pp. 33-83, 1989.

7. J. Cox, J. Ingersoll, and S. Ross, An Intertemporal General Equilibrium Model of Asset Prices, Econometrica 53, pp. 363-384, 1985a.

8. J. Cox, J. Ingersoll, and S. Ross, A Theory of Term Structure of Interest Rates, Econometrica 53, pp. 385-407, 1985 b.

9. C. Dellacherie and P. Meyer, Probabilities and Potential B: Theory of Martingales, NorthHolland Publishing Company, New York, 1982. 
10. J. Detemple and F. Zapatero, Asset Prices in An Exchange Economy with Habit Formation, Econometrica 59, pp. 1633-1658, 1991.

11. D. Duffie and L. Epstein, Stochastic Differential Utility and Asset Pricing, Econometrica, 1991, forthcoming.

12. K. Dunn and K. Singleton, Modeling the Term Structure of Interest Rates Under Nonseparability of Preferences and Durability of Goods, Journal of Financial Economics 17, pp. 27-55, 1986.

13. M. Eichenbaum and L. Hansen, Estimating Models with Intertemporal Substitution using Aggregate Time-Series Data, Journal of Business and Economic Statistics 8, 530069, 1990.

14. M. Eichenbaum, L. Hansen and K. Singleton, A Time Series Analysis of Representative Agent Models of Consumption and Leisure, Quarterly Journal of Economics CIII, pp. 51-78, 1988.

15. L. Epstein, A Simple Dynamic General Equilibrium Model, Journal of Economic Theory 41, pp.68-95, 1987.

16. L. Epstein and S. Zin, Substitution, Risk Aversion, and the Temporal Behavior of Consumption and Asset Returns: A Theoretical Framework, Econometrica 57, 1989.

17. IV. Fleming and R. Rishel, Deterministic and Stochastic Optimal Control, SpringerVerlag, 1975.

18. A. Gallant and G. Tauchen, Seminonparametric Estimation of Conditionally Constrained Heterogeneous Processes: Asset Pricing Applications, Econometrica 57, 1091-1120, 1989.

19. S. J, Grossman and G. Laroque, Asset Pricing and Optimal Portfolio Choice in the Presence of Illiquid Durable Consumption Goods, Econometrica 58, pp.25-51, 1990.

20. J.M. Harrison, Brownian Motion and Stochastic Flow Systems, John-Wiley \& Sons, Inc., 1985.

21. J. Heaton, An Empirical Investigation of Asset Pricing with Temporally Dependent Preference Specifications, unpublished manuscript, Sloan School of Management, MIT, 1990.

22. J. Heaton, The Interaction between Time-Nonseparable Preferences and Time Aggregation, unpublished manuscript, University of Chicago, 1988. 
23. A. Hindy and C. Huang, On Intertemporal Preferences for Uncertain Consumption: A Continuous Time Approach, Econometrica, 1991, forthcoming.

24. A. Hindy, C. Huang and D. Kreps, On Intertemporal Preferences in Continuous Time I: The Case of Certainty, Journal of Mathematical Economics, 1991, forthcoming.

25. J. Hotz, F. Kydland and G. Sedlacek, Intertemporal Preferences and Labor Supply, Econometrica 56, pp. 335-360, 1988.

26. T. Koopmans, Stationary Ordinal Utility and Impatience, Econometrica 28, pp. 287-309, 1960 .

27. N. Krylov, Controlled Diffusion Pracesses, Springer-Verlag, New York, 1980.

28. P.L. Lions and A. Sznitman, Stochastic Differential Equations with Reflecting Boundary Conditions, Communications on Pure and Applied Mathematics 37, pp. 511-537, 1984.

29. R. Merton, Optimum Consumption and Portfolio Rules in a Continuous Time Model, $J$. Econ. Theory 3, pp. 373-413, 1971.

30. S. Sundaresan, Intertemporally Dependent Preferences and the Volatility of Consumption and Wealth, Review of Financial Economics 2, pp.73-89, 1989.

31. H. Uzawa, Time Preferences, the Consumption Function and Optimum Asset Holdings, in Value, Capital and Growth: Papers in Honour of Sir John Hicks, (J, N. Wolfe, Ed.), Aldine, Chicago, 1968. 


\section{Appendix}

\section{A Proofs}

Proof of proposition 1 Let $C \in \mathcal{C}_{t}$ financed by $A \in \mathcal{A}_{t}$ be a feasible policy for (8). Let $W(s)$ and $z\left(s^{-}\right)$be the wealth and average past consumption at time $s \in[t, T]$ associated with $(C, A)$. Since wealth after consumption must be nonnegative, whenever the wealth reaches zero, the only feasible policy is to consume and invest zero afterwards.

Recall that $W$ has left-continuous and that $z$ has right-continuous sample paths. For simplicity of notation, we will denote $\hat{J}$ and its partial derivatives wit $\mathrm{h}$ respect to $W, z, S$ and time, evaluated at $\left(W(s), z\left(s^{-}\right), S(s), s\right)$, by $\hat{J}(s), \hat{J}_{W}(s), \hat{J}_{z}(s), \hat{J}_{S}(s), \hat{J}_{s}(s)$, respectively.

Note that, given the admissibility condition (6) and the growth condition on $u$ given in (17), we know

$$
E_{t}\left[\int_{t}^{T}|u(z(s), s)| d t\right]<\infty .
$$

Define $\varrho=\inf \{s \geq t: W(s)=0\}$, where we have used the convention that if the infimum does not exist, we set it to be $T .^{14}$ Also, let the points of discontinuity of $C(\omega, s)$ on the stochastic interval $[t, \varrho)$ be $t_{1}(\omega), t_{2}(\omega), \ldots$

Fix $\tau \in(t, T)$. Generalized Itô's lemma implies that

$$
\begin{aligned}
& \int_{t}^{\varrho \wedge \tau} u(z(s), s) d s+\hat{J}\left(W(\varrho \wedge \tau), z\left((\varrho \wedge \tau)^{-}\right), S(\varrho \wedge \tau), \varrho \wedge \tau\right) \\
& \quad=\int_{t}^{\varrho \wedge \tau} u(z(s), s) d s+\hat{J}\left(W(t), z\left(t^{-}\right), S(t), t\right)+\int_{t}^{\varrho \wedge \tau}\left[\mathcal{D}^{A} \hat{J}(s)+\hat{J}_{s}(s)\right] d s \\
& +\int_{t}^{\varrho \wedge \tau}\left[\hat{J}_{W}(s) W(s) A(s)^{\top} I_{S^{-1}}(s) \sigma(s)+\hat{J}_{S}(s)^{\top} \sigma(s)\right] d B(s)+\int_{t^{-}}^{(\varrho \wedge \tau)^{-}}\left[\beta \hat{J}_{z}(s)-\hat{J}_{W^{\prime}}(s)\right] d C(s) \\
& +\sum_{i}\left[\hat{J}\left(t_{i}^{+}\right)-\hat{J}\left(t_{i}\right)\right]-\sum_{i}\left[\hat{J}_{W}\left(t_{i}\right) \Delta W\left(t_{i}\right)+\hat{J}_{z}\left(t_{i}\right) \Delta z\left(t_{i}\right)\right] \quad a . s .
\end{aligned}
$$

where $\varrho \wedge \tau$ denotes $\min [\varrho, \tau]$. By the hypothesis that $\hat{J}$ is concave in its first two arguments, we know that,

$$
\begin{aligned}
\hat{J}\left(t_{i}^{+}\right)-\hat{J}\left(t_{i}\right) & \leq \hat{J}_{W}\left(t_{i}\right) \Delta W\left(t_{i}\right)+\hat{J}_{z}\left(t_{i}\right) \Delta z\left(t_{i}\right) \\
& =-\hat{J}_{W}\left(t_{i}\right) \Delta C\left(t_{i}\right)+\hat{J}_{z}\left(t_{i}\right) \beta \Delta C\left(t_{i}\right) \quad \forall t_{i} \quad \text { a.s. }
\end{aligned}
$$

\footnotetext{
${ }^{14} \varrho$ is an optional time in that, $\{\omega \in \Omega: \varrho(\omega) \leq t\} \in \mathcal{F}_{s} \forall t \in[0, T]$.
} 
Thus,

$$
\begin{aligned}
& \int_{t}^{\varrho \wedge \tau} u(z(s), s) d s+\hat{J}\left(W(\varrho \wedge \tau), z\left((\varrho \wedge \tau)^{-}\right), S(\varrho \wedge \tau), \varrho \wedge \tau\right) \\
& \leq \hat{J}\left(W(t), z\left(t^{-}\right), S(t), t\right)+\int_{t}^{e^{\wedge \tau}}\left[\hat{J}_{W}(s) W(s) A(s)^{\top} I_{S^{-1}}(s) \sigma(s)+\hat{J}_{S}(s)^{\top} \sigma(s)\right] d B(s)
\end{aligned}
$$

where we have used the fact that $\hat{J}$ satisfies the differential inequality and $C$ is increasing.

Now define

$$
\varrho_{n}=\inf \left\{s \leq \varrho \wedge \tau: \int_{t}^{s}\left[\hat{J}_{W}(s) W(s) A(s)^{\top} I_{S^{-1}}(s) \sigma(s)+\hat{J}_{S}(s)^{\top} \sigma(s)\right]^{2} d s=n\right\}
$$

From the application of the generalized Itô's lemma in (60), we know that

$$
\int_{t}^{\varrho \wedge \tau}\left[\hat{J}_{W}(s) W(s) A(s)^{\top} I_{S^{-1}}(s) \sigma(s)+\hat{J}_{S} S(s)^{\top} \sigma(s)\right]^{2} d s<\infty \quad \text { a.s. },
$$

and, hence, we conclude that $\varrho_{n} \uparrow \varrho \wedge \tau$ a.s. On the stochastic interval $\left[t, \varrho_{n}\right]$,

$$
\int_{t}^{s}\left[\hat{J}_{W}(v) W(v) A(v)^{\top} I_{S^{-1}}(v) \sigma(v)+\hat{J}_{S}(v)^{\top} \sigma(v)\right] d B(v)
$$

is a martingale; see, for example, Liptser and Shiryayev (1977, chapter 4).

Replacing $\varrho \wedge \tau$ of $(61)$ by $\varrho_{n}$ and taking expectation gives

$$
E_{t}\left[\int_{t}^{\varrho_{n}} u(z(s), s) d s+\hat{J}\left(W\left(\varrho_{n}\right), z\left(\varrho_{n}^{-}\right), S\left(\varrho_{n}\right), \varrho_{n}\right)\right] \leq \hat{J}\left(W(t), z\left(t^{-}\right), S(t), t\right) .
$$

Note that (59) and the fact that $\varrho_{n} \rightarrow \varrho \wedge \tau$ a.s. imply

$$
E_{t}\left[\int_{t}^{\rho_{n}} u(z(s), s) d s\right] \rightarrow E_{t}\left[\int_{t}^{\rho \wedge \tau} u(z(s), s) d s\right]
$$

by Lebesgue dominated convergence theorem. We want to show that

$$
E_{t}\left[\hat{J}\left(W\left(\varrho_{n}\right), z\left(\varrho_{n}^{-}\right), S\left(\varrho_{n}\right), \varrho_{n}\right)\right] \rightarrow E_{t}\left[\hat{J}\left(W(\varrho \wedge \tau), z\left((\varrho \wedge \tau)^{-}\right), S(\varrho \wedge \tau), \varrho \wedge \tau\right)\right]
$$

as $n \rightarrow \infty$. In our current context, this conclusion follows from Lebesgue theorem by the leftcontinuity of the sample paths of $\left(W(s), z\left(s^{-}\right), S(s)\right)$, the growth condition on $\hat{J}$ in $(17)$ and the moment conditions on $W$ and on $C$ in (6); see, for example, Fleming and Rishel (1975, theorem V.5.1).

The assertion then follows from letting $\tau \uparrow T$, from boundary conditions of $\hat{J}$ at $T$ and at $W=0$, and from the fact that $V$ is increasing and $W\left(T^{+}\right) \leq W(T)$. 
ProOF OF THEOREM 1. The generalized Itô's lemma allows us to write

$$
\begin{aligned}
& \mathrm{E}_{t}\left[\int_{t}^{\varrho} u\left(z^{*}(s), s\right) d s+\hat{J}\left(W^{*}(\varrho), z^{*}\left(\varrho^{-}\right), S(\varrho), \varrho\right)\right] \\
= & \mathrm{E}_{t}\left[\int_{t}^{\varrho} u\left(z^{*}(s), s\right) d s+\hat{J}\left(W^{*}\left(t^{+}\right), z^{*}(t), S(t), t\right)\right. \\
& \left.\left.+\int_{t}^{T}\left[\hat{J}_{W}(s) W^{*}(s) A^{*}(s)^{\top} I_{S^{-1}}(s) \sigma(s)+\hat{J}_{S}(s)^{\top} \sigma(s)\right] d B(s)\right]\right] \\
= & \hat{J}\left(W^{*}\left(t^{+}\right), z^{*}(t), S(t), t\right)=\hat{J}\left(W^{*}(t), z^{*}\left(t^{-}\right), S(t), t\right),
\end{aligned}
$$

where the first equality follows from (19), (20) and (21), the second equality follows from the identical arguments used to prove Proposition 1, and the third equality follows from (21). Using the boundary conditions of (15) and (16) allows us to conclude that $\hat{J}=J$ and thus $\left(C^{*}, A^{*}\right)$ is an optimal policy.

ProOF OF THEOREM 2. For any finite $T$, using identical arguments as in proving Theorem 1 we get,

$$
\hat{J}\left(W(t), z\left(t^{-}\right), S(t), t\right) \geq E_{t}\left[\int_{t}^{T} u(z(s), s) d s+\hat{J}\left(W(T), z\left(T^{-}\right), S(T), T\right)\right] .
$$

Since $\hat{J}$ is positive, we can drop the second term on the right-hand side of the above relation and still maintain the inequality. Since the inequality holds for all $T>t$. We can let $T \rightarrow \infty$ and conclude that $\hat{J} \geq J$.

Then the assertion follows from arguments identical to those used to prove Theorem 1 in addition to using condition (28). 


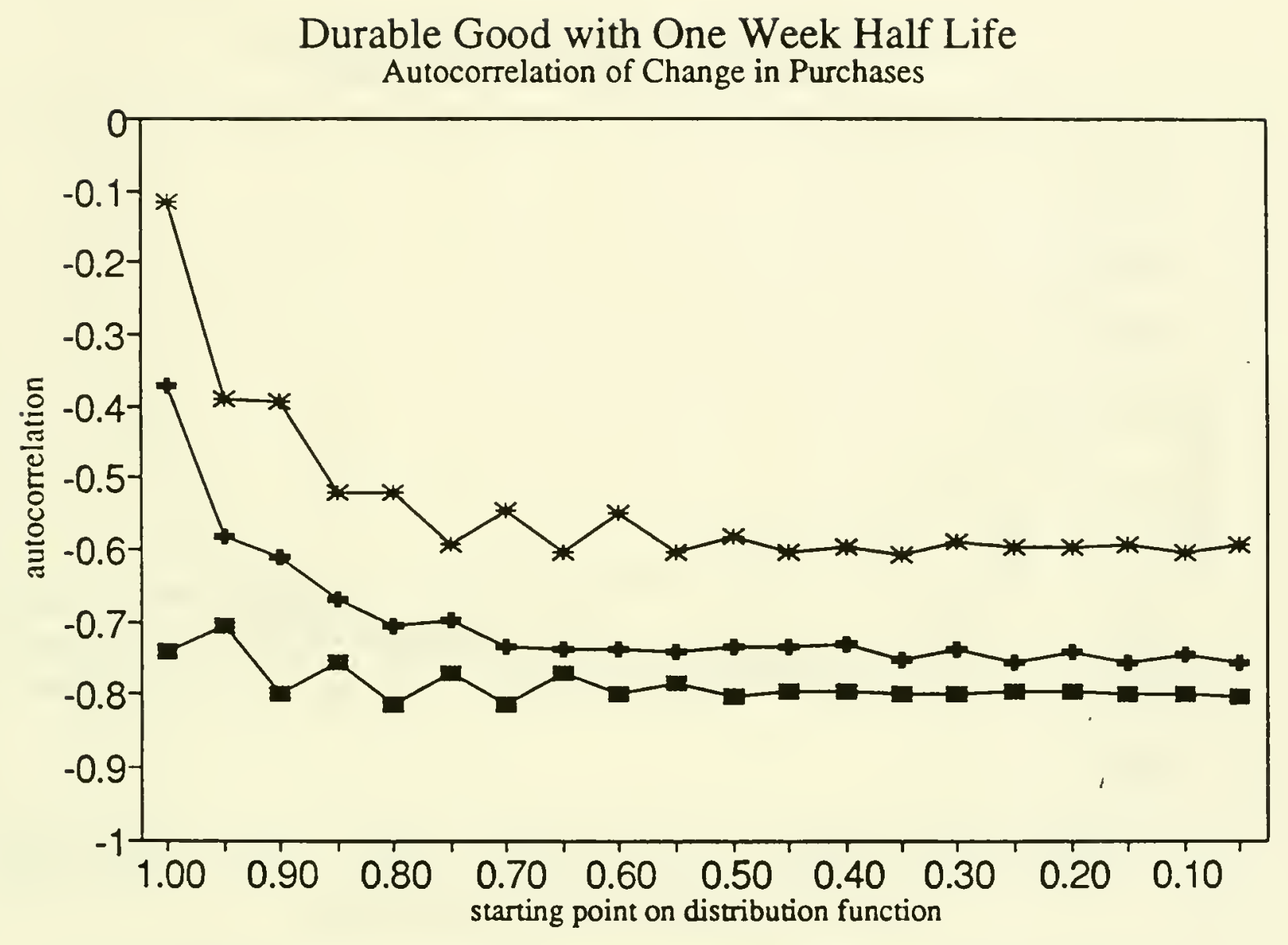

Measurement frequency $\rightarrow$ weekly $\rightarrow-$ monthly $\rightarrow$ quarterly 


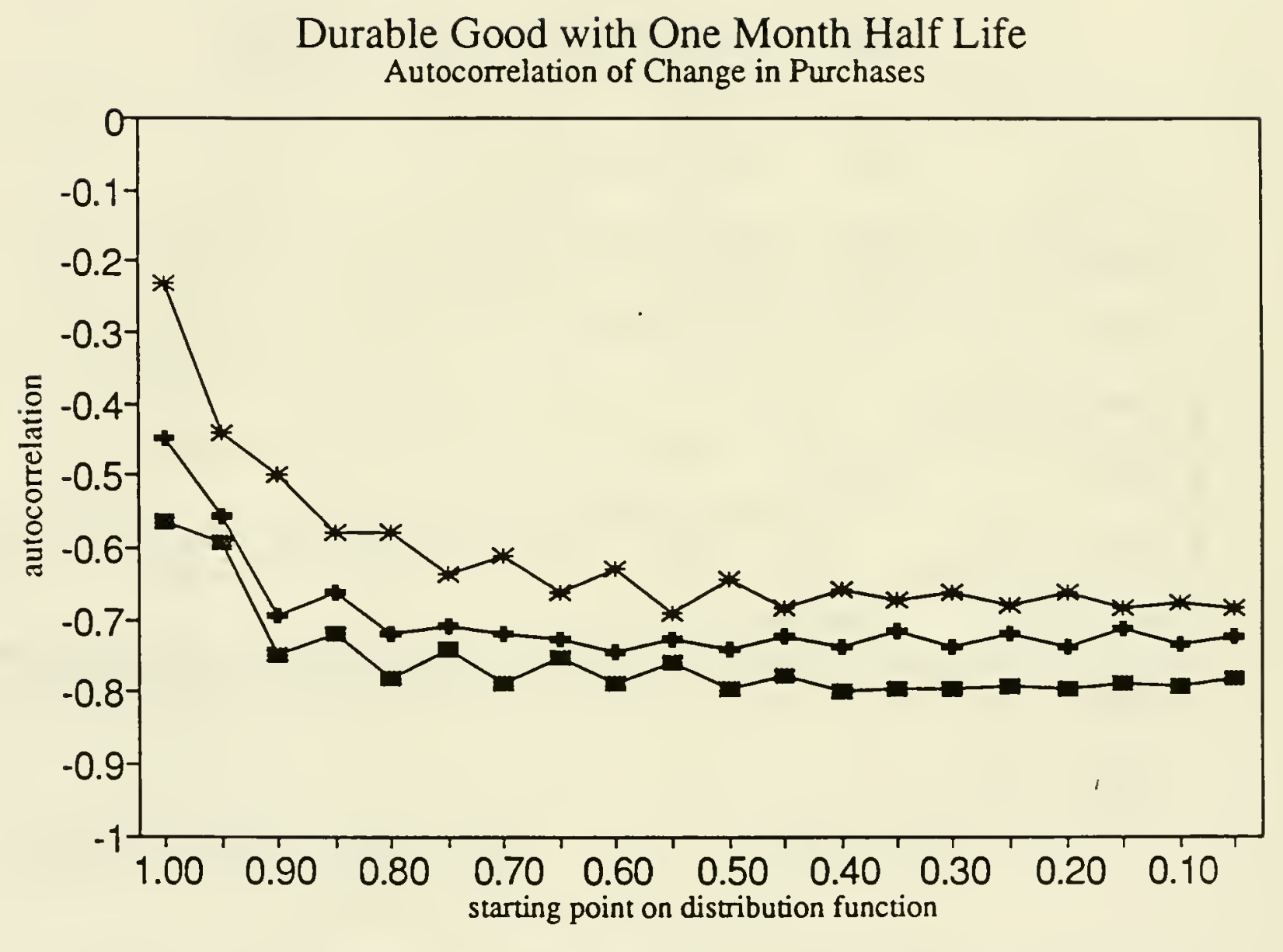

Measurement frequency $\rightarrow-$ weekly $\rightarrow$ monthly $\rightarrow-$ quarterly 


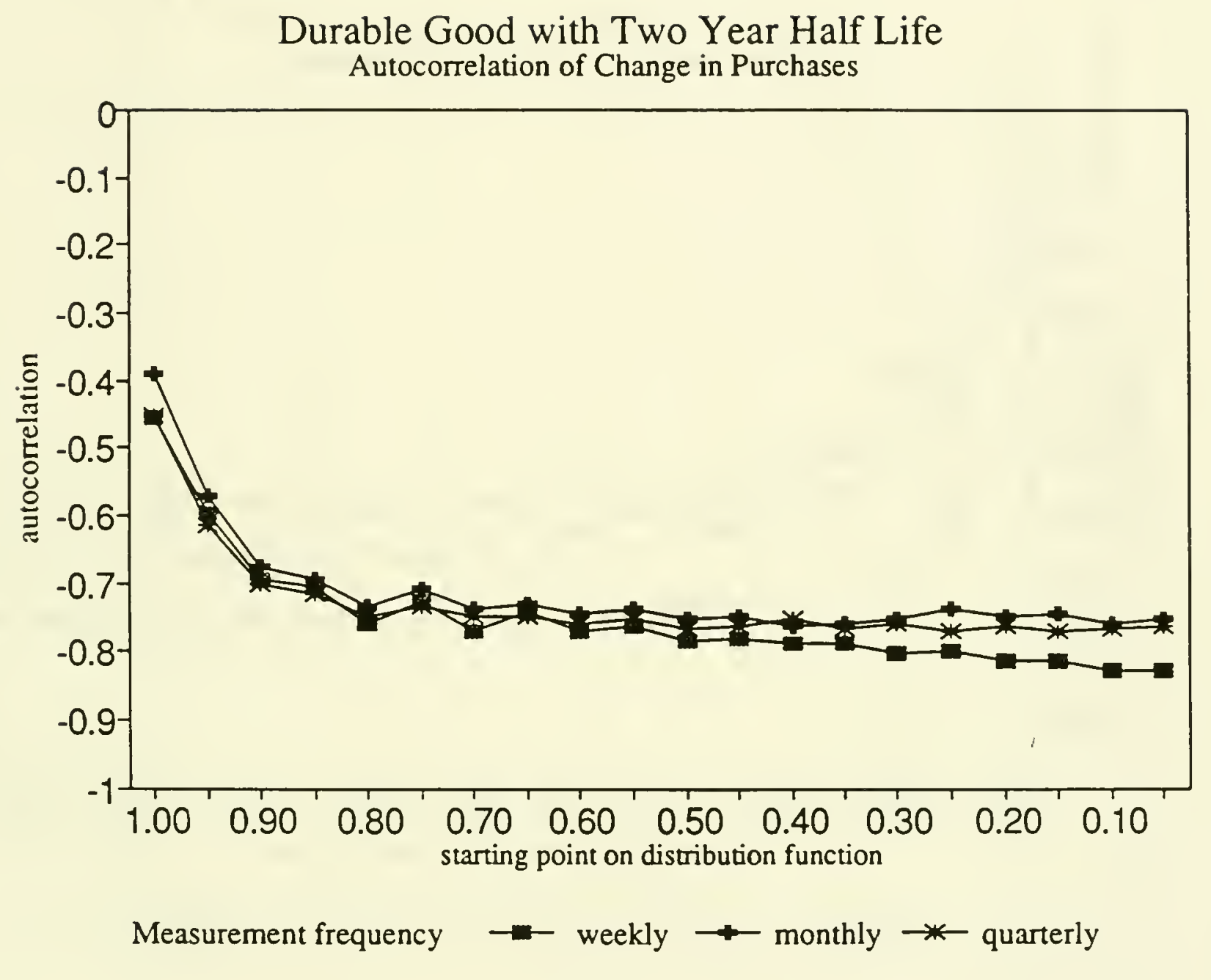

Figure 3 


\section{Quarterly Purchases of the Durable Good Autocorrelation of Change in Purchases}

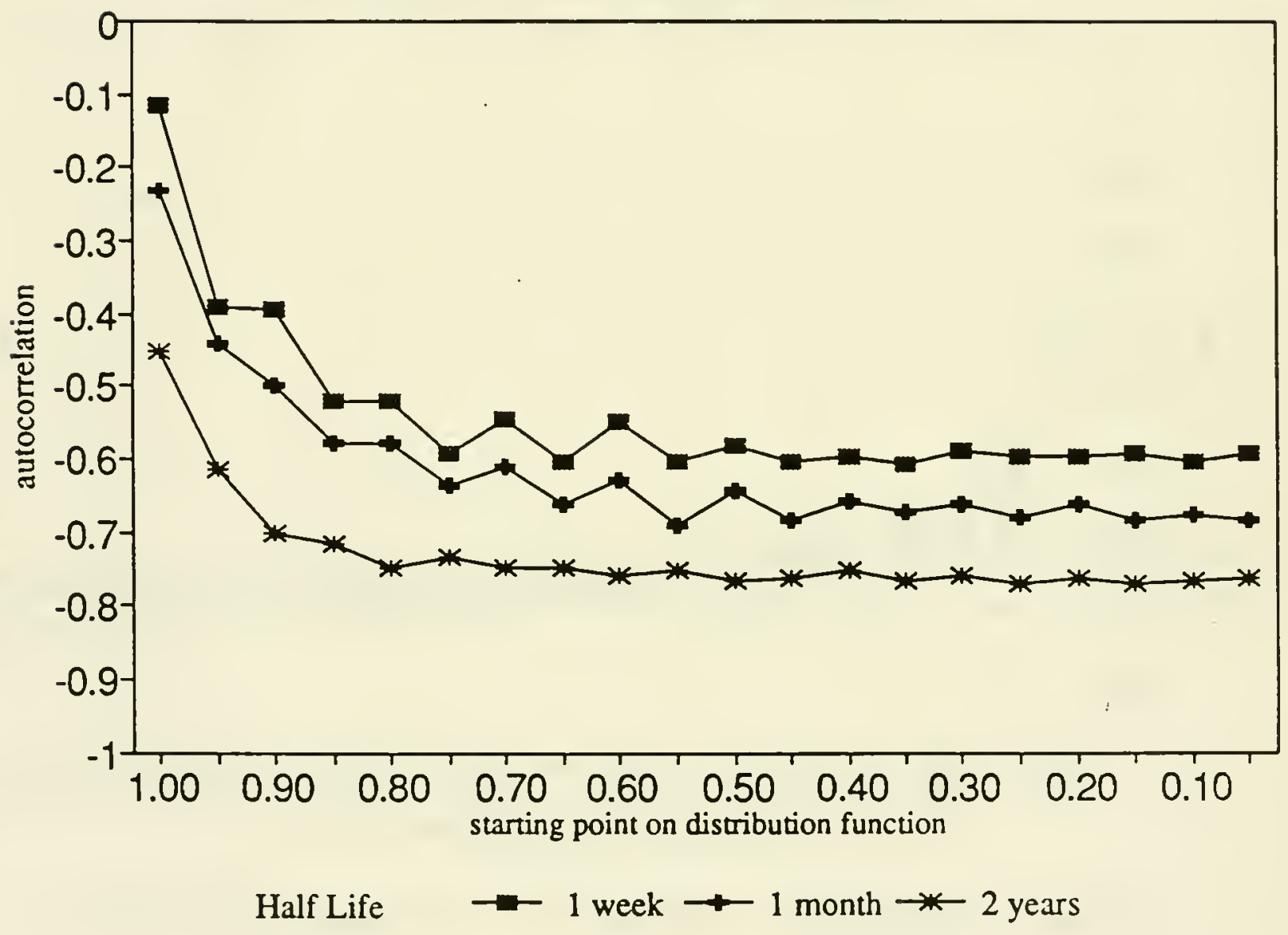

Figure 4 


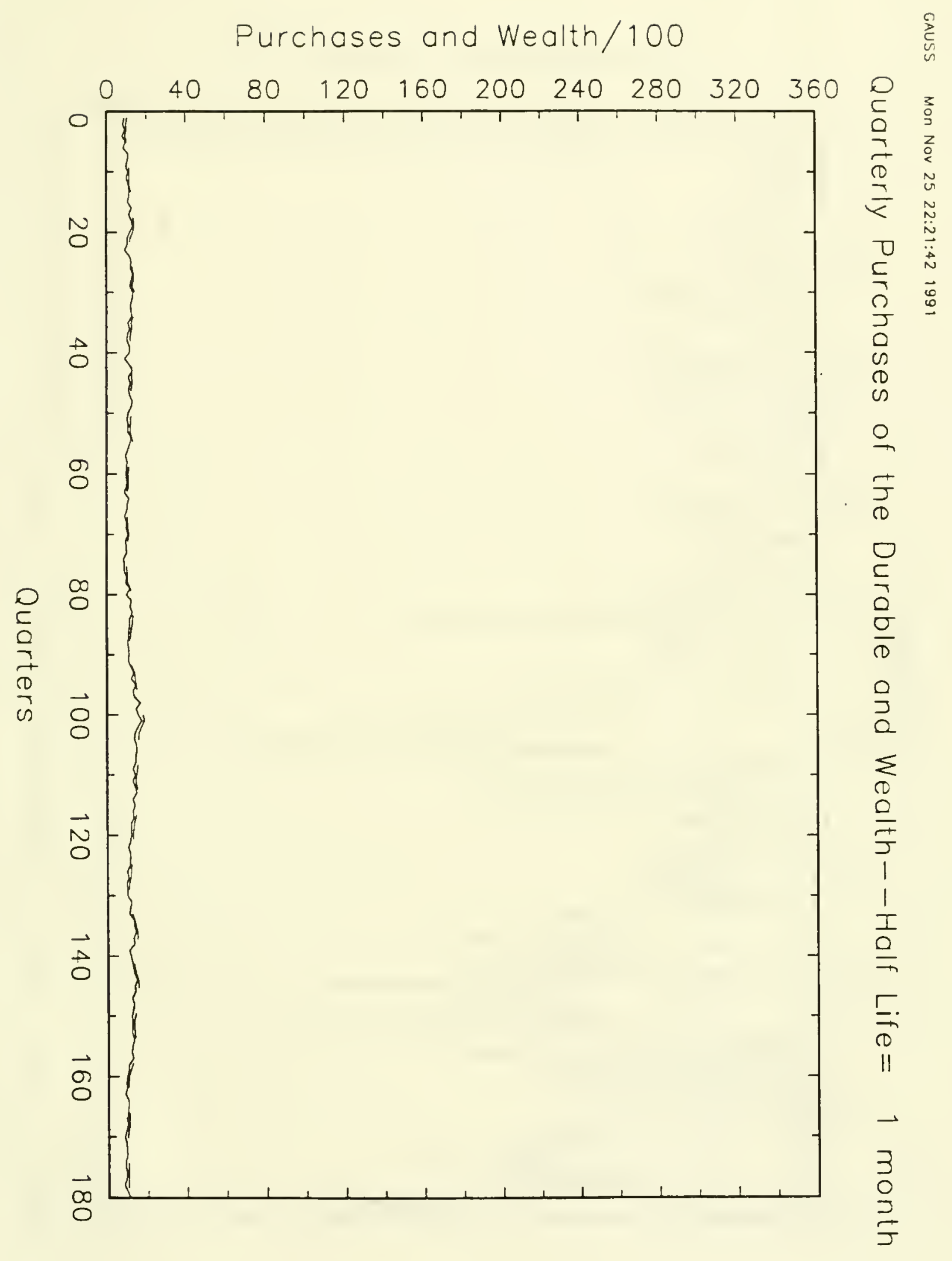

Eigure 5 


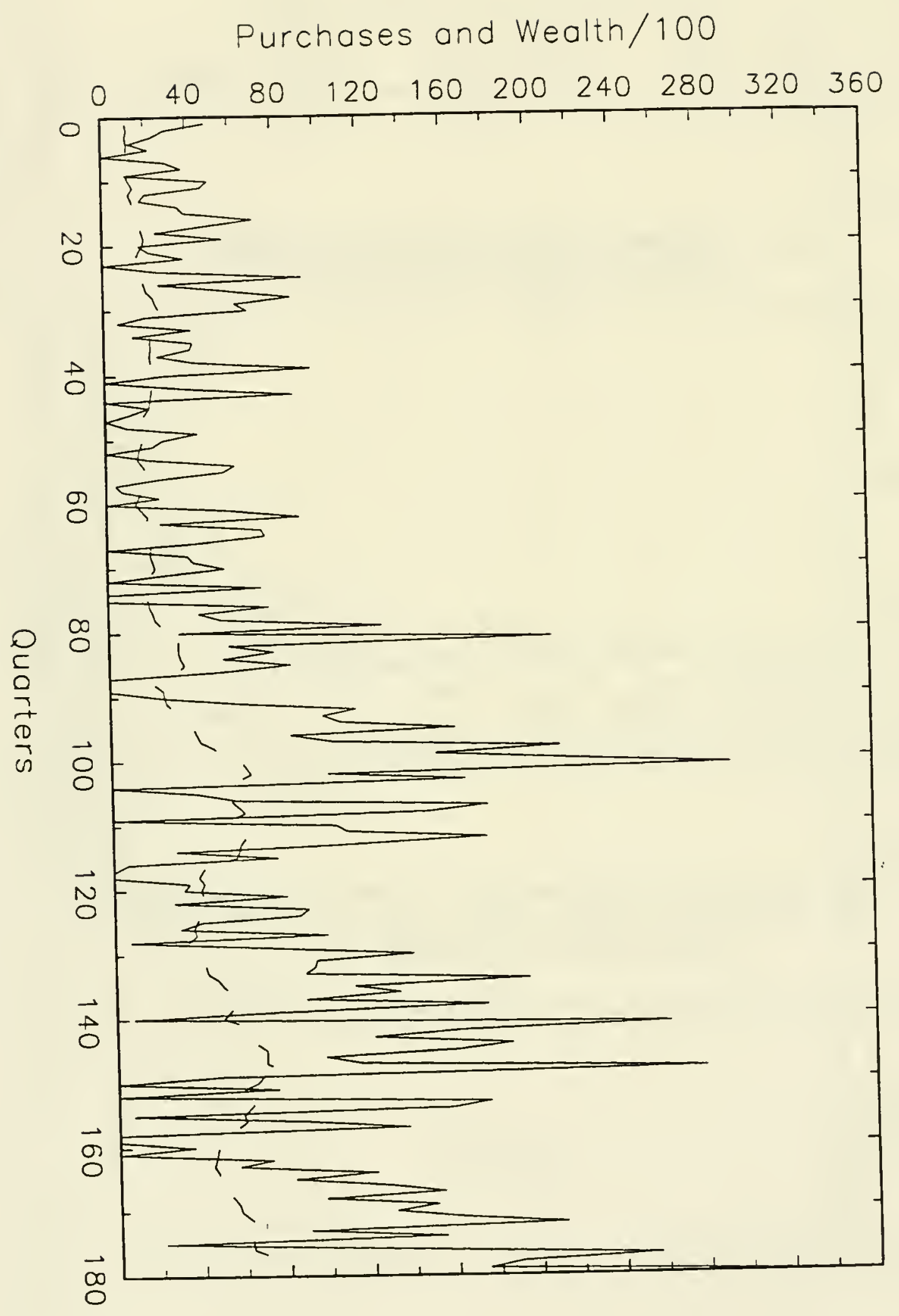

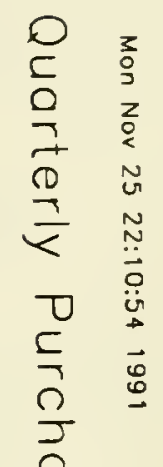

(D)

o

$\underline{1}$

5

$\square$

궁

$\frac{\sigma}{0}$

0

3

ㅇ

$\sum$

(1)

0

J

I

1

픙

E.

(D)

II

$N$
$k$
0
0
0

Figure 6 




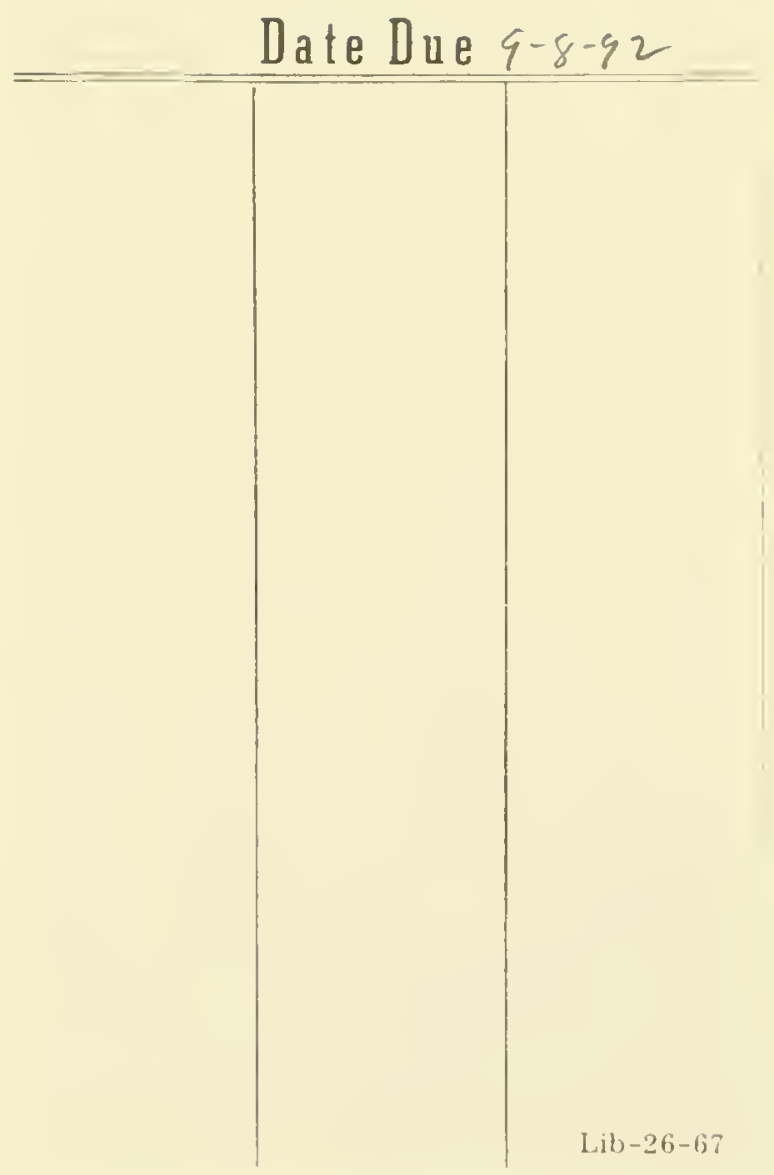




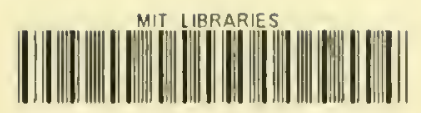

3 9080007333708 
